\title{
Pacific
}

Journal of

Mathematics

\section{DIRAC COHOMOLOGY OF WALLACH REPRESENTATIONS}

Jing-Song Huang, Pavle Pandžić and Victor Protsak 


\title{
DIRAC COHOMOLOGY OF WALLACH REPRESENTATIONS
}

\author{
Jing-Song Huang, PAVle PAndžić And Victor ProtsaK
}

Let $G$ be either the metaplectic double cover of $\operatorname{Sp}(2 n, \mathbb{R})$, or $\mathrm{SO}^{*}(2 n)$, or $\mathrm{SU}(p, q)$. Let $\mathfrak{g}$ be the complexified Lie algebra of $G$ and let $K$ be a maximal compact subgroup of $G$. Let $X$ be one of the Wallach modules for the pair $(\mathfrak{g}, K)$. In other words, $X$ corresponds to a discrete point in the classification of unitary lowest weight modules with scalar lowest $K$ type. The purpose of this paper is to calculate the Dirac cohomology of $X$. Our approach is based on the explicit knowledge of the $K$-types of $X$. We establish a bijection between certain $K$-types $E_{i}$ of $X$ and certain $\tilde{K}$ types $F_{i}$ of the spin module, where $\tilde{K}$ is the spin double cover of $K$. The Dirac cohomology is then realized as the set of Parthasarathy-Ranga-RaoVaradarajan components of $E_{i} \otimes F_{i}$.

\section{Introduction}

Let $G$ be a connected real reductive Lie group with a Cartan involution $\Theta$. We assume that the group of fixed points of $\Theta, K=G^{\Theta}$, is a maximal compact subgroup of $G$. We will denote by $\mathfrak{g}=\mathfrak{k} \oplus \mathfrak{p}$ the Cartan decomposition of the complexified Lie algebra of $G$. The classification of the unitary dual of $G$ is a significant open problem in representation theory. An important necessary condition for unitarity (unitarizability) of a simple Harish-Chandra module $X$, due to Parthasarathy [1980], is the Dirac inequality (Proposition 3.3), a byproduct of studying the action of the Dirac operator $D$ on $X \otimes S$ where $S$ is a spin module for the Clifford algebra $C(\mathfrak{p})$ ([Parthasarathy 1972; Vogan 1997]; see Section 3 for details.) If $X$ is unitary, then $D$ is Hermitian (self-adjoint) with respect to a natural Hermitian inner product, and hence $D^{2} \geq 0$ on $X \otimes S$. Writing this inequality more explicitly leads directly to the Dirac inequality.

While the Dirac inequality is necessary for unitarity, it is by no means sufficient. A careful analysis of this fact led Vogan [1997] to the notion of Dirac cohomology

Huang is supported by research grant 600807 and 601609 from the Research Grant Council of HKSAR, China. Pandžić is supported by a grant from the Ministry of Education, Science and Sport of the Republic of Croatia. Pandžić and Protsak thank the Mathematics Department of Cornell University for excellent working conditions under which part of this work was done.

MSC2000: 22E46.

Keywords: reductive Lie group, unitary representation, Dirac cohomology, Wallach representation. 
(see also [Huang and Pandžić 2002]). The Dirac cohomology $H_{D}(X)$ of $X$ is $\operatorname{Ker} D$ divided by the intersection $\operatorname{Im} D \cap \operatorname{Ker} D$. It is a module for the spin double cover $\tilde{K}$ of $K$. If $X$ is unitary, we have $H_{D}(X)=\operatorname{Ker} D=\operatorname{Ker} D^{2}$, since $D$ is Hermitian. In particular, the unitary $X$ for which the equation $D^{2}=0$ has nonzero solutions in $X \otimes S$ are precisely the ones with nonzero Dirac cohomology. These representations are extremal in the sense that Dirac inequality becomes equality on some of the $\tilde{K}$-types of $X \otimes S$. Once such a $\tilde{K}$-type $E$ is fixed, there are only a few possible irreducible modules $X$ with $H_{D}(X) \supseteq E$. Namely, the main result of [Huang and Pandžić 2002], conjectured by Vogan, says that such $E$ determines the infinitesimal character of $X$.

The Dirac cohomology of various classes of representations turned out to be intimately related with several classical subjects of representation theory like characters and the construction of the discrete series [Huang and Pandžić 2006]. It is also related to nilpotent Lie algebra cohomology [Huang et al. 2006] and to $(\mathfrak{g}, K)$ cohomology [Huang et al. 2009; Pandžić 2004].

Modules with nonzero Dirac cohomology include finite-dimensional modules with highest weight stable under the Cartan involution [Kostant 1999; 2003; Huang et al. 2009]. Further examples are $A_{\mathfrak{q}}(\lambda)$ modules with $\lambda$ stable under the Cartan involution and sufficiently regular [Huang et al. 2009]; this class of modules includes the discrete series.

Another class of modules known to have nonzero Dirac cohomology are the unitary highest (or lowest) weight modules. Their Dirac cohomology is in principle known by results in [Huang et al. 2006] and [Enright 1988]. In the first of these papers we showed that the Dirac cohomology is in some sense equal to the $\mathfrak{p}^{+}$ cohomology, which was in turn determined by Enright. (Recall that the $K$-module $\mathfrak{p}$ breaks up as $\mathfrak{p}^{+} \oplus \mathfrak{p}^{-}$since the pair $(\mathfrak{g}, \mathfrak{k})$ is now Hermitian symmetric.) Enright's description is quite complicated and abstract; but in [Huang et al. 2006] we showed that the Dirac cohomology can be viewed as a space of harmonic representatives of the $\mathfrak{p}^{+}$cohomology, so one can hope for a more explicit and concrete description.

The goal of this paper is to determine the Dirac cohomology of certain unitary lowest weight modules as directly and explicitly as possible. The modules we consider are the Wallach representations of the symplectic, orthogonal and indefinite unitary groups. Wallach modules are named after Nolan Wallach, who constructed them in the algebraic setting [1979]; they were recovered in the analytic setting in [Vergne and Rossi 1976]. ${ }^{1}$ Together with the trivial module, Wallach modules form the discrete part in the classification of all unitary lowest weight modules with scalar lowest $K$-type [Enright et al. 1983].

The lowest weights of Wallach modules are of the form $c k \zeta$. Here $k$ is an integer between 1 and $r-1$, where $r$ is the split rank (real rank) of $G$. The constant $c$ is

\footnotetext{
${ }^{1}$ Wallach's priority is confirmed in the introduction of Vergne and Rossi's paper.
} 
$\frac{1}{2}$ in the symplectic case, 2 in the orthogonal case and 1 in the unitary case, while $\zeta$ is the fundamental weight of $\mathfrak{g}$ orthogonal to the roots of $\mathfrak{k}$.

The Wallach representations are also important in invariant theory and the theory of reductive dual pairs [Howe 1989; 1995]. These modules can be realized as theta lifts of the trivial representation in compact dual pair correspondences. Furthermore, they arise in the study of the geometry of nilpotent orbits [Nishiyama et al. 2001, Section 7]. They are also important in another approach to the classification of unitary lowest weight modules [Adams 1987]: they are the basic ones from which all others can be obtained by cohomological induction. Finally, they appear in mathematical physics: they are related to the generalized hydrogen atom and to generalized MICZ-Kepler problems [Meng 2008; 2007; 2010].

We note that the continuous family of unitary highest weight modules are full generalized Verma modules. As such they are also $A_{\mathfrak{q}}(\lambda)$-modules for the maximal parabolic subalgebra $\mathfrak{q}=\mathfrak{k} \oplus \mathfrak{p}^{-}$of $\mathfrak{g}$. Therefore their Dirac cohomology can be calculated using the methods and results of [Huang et al. 2009]. This is another reason we focus our consideration on Wallach representations.

In the symplectic case, together with each Wallach module $V_{k}^{+}(k=1, \ldots, r-1$, where $r$ is the real rank of $G$ ), we study another unitary lowest weight module $V_{k}^{-}$, with nonscalar lowest $K$-type. For example, the even half of the oscillator representation is the Wallach module $V_{1}^{+}$, and the odd half is $V_{1}^{-}$. Studying $V_{k}^{-}$ together with $V_{k}^{+}$requires no additional work, and moreover, replacing the Wallach module with $V_{k}=V_{k}^{+} \oplus V_{k}^{-}$makes our main result stated below more uniform. We provide a detailed description of Wallach representations, as well as of the modules $V_{k}^{-}$in the symplectic case, in Section 2.

Let us introduce some standard notation. Let $t$ be the common Cartan subalgebra of $\mathfrak{g}$ and $\mathfrak{k}$. We choose positive roots for $\mathfrak{g}$ and $\mathfrak{k}$ with respect to $\mathfrak{t}$ in such a way that

$$
\Delta^{+}(\mathfrak{g}, \mathfrak{t})=\Delta^{+}(\mathfrak{k}, \mathfrak{t}) \cup \Delta\left(\mathfrak{p}^{+}\right),
$$

where $\Delta\left(\mathfrak{p}^{+}\right)$denotes the set of $\mathfrak{t}$-weights of $\mathfrak{p}^{+}$. As usual, we denote by $\rho$ the half sum of roots in $\Delta^{+}(\mathfrak{g}, \mathfrak{t})$, by $\rho_{c}$ the half sum of roots in $\Delta^{+}(\mathfrak{k}, \mathfrak{t})$, and by $\rho_{n}$ the half-sum of roots in $\Delta\left(\mathfrak{p}^{+}\right)$. Let $W_{G}$ and $W_{K}$ be the Weyl groups of $(\mathfrak{g}, \mathfrak{t})$ and $(\mathfrak{k}, \mathfrak{t})$ respectively. For any $\mathfrak{k}$-dominant weight $\mu \in \mathfrak{t}^{*}$, we denote by $E_{\mu}$ the irreducible finite-dimensional $\mathfrak{k}$-module with highest weight $\mu$.

We are now ready to state our main result.

Theorem 1.2. Let $G$ be the metaplectic double cover of $\operatorname{Sp}(2 n, \mathbb{R})$; or $\mathrm{SO}^{*}(2 n)$; or $\mathrm{SU}(p, q)$. Let $X$ be one of the Wallach modules for the corresponding pair $(\mathfrak{g}, K)$, or in the symplectic case, the direct sum of the Wallach module $V_{k}^{+}$with the associated module $V_{k}^{-}$. Denote by $\Lambda \in \mathfrak{t}^{*}$ the $\mathfrak{g}$-dominant representative of the 
infinitesimal character of $X$. If $\Lambda_{1}, \ldots, \Lambda_{m}$ are the $W_{G}$-translates of $\Lambda$ which are dominant and regular for $\mathfrak{k}$, then

$$
H_{D}(X)=\bigoplus_{i=1}^{m} E_{\Lambda_{i}-\rho_{c}} .
$$

In the rest of the introduction we explain our strategy for proving Theorem 1.2 and comment on its extensions. It will become clear from general results about Dirac cohomology that any irreducible $\tilde{K}$-submodule of $H_{D}(X)$ must be of the form $E_{\Lambda_{i}-\rho_{c}}$. Thus, our task will be to show that each $E_{\Lambda_{i}-\rho_{c}}$, in fact, appears with multiplicity one. In order to do this, we will first demonstrate in Proposition 3.4 that any $\tilde{K}$-submodule of $X \otimes S$ appearing in $H_{D}(X)$ must be the ParthasarathyRanga-Rao-Varadarajan (PRV) component of the tensor product of a $K$-type of $X$ and a $\tilde{K}$-type of the spin module $S$. The rest of the paper is then devoted to case-by-case calculations showing that for each $i$, there is in fact a unique $K$-type $E_{\mu_{i}} \subset X$ and a unique $\tilde{K}$-type $E_{\sigma_{i}} \subset S$ such that $E_{\Lambda_{i}-\rho_{c}}$ is the PRV component of $E_{\mu_{i}} \otimes E_{\sigma_{i}}$.

We will determine $\mu_{i}$ and $\sigma_{i}$ very explicitly from the shortest element $w_{i}$ of $W_{G}$ such that $w_{i} \Lambda=\Lambda_{i}$. We denote by $W_{X}$ the set of all $w_{i}$ for $i=1, \ldots, m$. We will prove the following version of Theorem 1.2.

Theorem 1.3. In the setting of Theorem 1.2, let $W_{X}$ be the collection of the shortest element $w_{i}$ of $W_{G}$ such that $w_{i} \Lambda=\Lambda_{i}$. Then

$$
H_{D}(X)=\bigoplus_{w \in W_{X}} E_{w \Lambda-\rho_{c}} .
$$

Moreover, for each $w \in W_{X}$ there is a unique $K$-type $E_{\mu(w)}$ of $X$, appearing in $X$ with multiplicity one, and a unique $\tilde{K}$-type $E_{\sigma(w)}$ of $S$, such that $E_{w \Lambda-\rho_{c}}$ is the $P R V$ component of $E_{\mu(w)} \otimes E_{\sigma(w)}$.

In each of the cases we are considering, all ingredients of Theorem 1.3 will be made completely explicit. See Theorems 4.3, 5.2 and 7.6. We actually prefer the formulation of Theorem 1.3 because in other known cases, Dirac cohomology is usually expressed as a sum over a subset of the Weyl group.

Finally, we note that there are several other Hermitian cases which are not considered in this paper: the cases of $O(2, m)$ and the exceptional cases E III and E VII [Enright et al. 1983]. In each of these cases we have obtained a result analogous to Theorem 1.3. Note that there is only one Wallach module for $O(2, m)$, which can be constructed using the noncompact dual pair $\operatorname{Sp}(2, \mathbb{R}) \times O(p, 2)$ in $\operatorname{Sp}(2(p+2), \mathbb{R})$. It is the theta-lift of the trivial representation of $\operatorname{Sp}(2, \mathbb{R})$. This is a special case of theta-lifting for the dual pair $\operatorname{Sp}(2 k, \mathbb{R}) \times O(p, q)$ in $\operatorname{Sp}(2 k(p+q), \mathbb{R})$, considered in [Zhu and Huang 1997]. There is one Wallach 
module for the exceptional case E III and two Wallach modules for E VII. We do not include these cases here to keep this paper to a reasonable size and we do plan to consider them together with the general cases of noncompact dual pairs listed in [Nishiyama et al. 2001, Section 3, Table 1].

\section{A description of Wallach representations}

Let $\mathfrak{g}_{0}$ be one of the simple real Lie algebras $\mathfrak{s p}(2 n, \mathbb{R}), \mathfrak{s o} *(2 n, \mathbb{R})$ or $\mathfrak{s u}(p, q)$. Let $\mathfrak{g}_{0}=\mathfrak{k}_{0} \oplus \mathfrak{p}_{0}$ be a Cartan decomposition of $\mathfrak{g}_{0}$, and let $\mathfrak{g}=\mathfrak{k} \oplus \mathfrak{p}$ be the complexified Cartan decomposition. Since the pair $(\mathfrak{g}, \mathfrak{k})$ is Hermitian, $\mathfrak{p}$ decomposes as $\mathfrak{p}^{+} \oplus \mathfrak{p}^{-}$ as a $\mathfrak{k}$-module, and we fix such a decomposition.

As in the introduction, let $\mathfrak{t}$ be a common Cartan subalgebra for $\mathfrak{g}$ and $\mathfrak{k}$. We choose systems of positive roots for $\mathfrak{g}$ and $\mathfrak{k}$ with respect to $\mathfrak{t}$ so that (1.1) holds. Below we will make explicit choices in each of the three cases.

Let $\zeta$ be the fundamental weight of $\mathfrak{g}$ which is orthogonal to all the roots of $\mathfrak{k}$. Furthermore, let $c=\frac{1}{2}$ if $\mathfrak{g}_{0}=\mathfrak{s p}(2 n, \mathbb{R}), c=2$ if $\mathfrak{g}_{0}=\mathfrak{s o}^{*}(2 n, \mathbb{R})$ and $c=1$ if $\mathfrak{g}_{0}=\mathfrak{s u}(p, q)$. Finally, let $r$ be the split rank (real rank) of $\mathfrak{g}_{0}$. In other words, $r$ is the dimension of a maximal abelian subspace of $\mathfrak{p}_{0}$. It is well known (see [Knapp 2002, p. 107], for example) that $r=n$ if $\mathfrak{g}_{0}=\mathfrak{s p}(2 n, \mathbb{R}), r=[n / 2]$ if $\mathfrak{g}_{0}=\mathfrak{s o}^{*}(2 n, \mathbb{R})$ and $r=\min (p, q)$ if $\mathfrak{g}_{0}=\mathfrak{s u}(p, q)$.

For an integer $k$ such that $1 \leq k<r$, the $k$-th Wallach representation is the unitary lowest weight representation with lowest weight $k c \zeta$. This definition is taken from [Enright and Willenbring 2004, 1.4] except that they consider highest and not lowest weight modules, which corresponds to exchanging the roles of $\mathfrak{p}^{+}$and $\mathfrak{p}^{-}$ and introducing a minus sign on the weights. The same highest weight modules are described in a slightly different fashion in [Enright et al. 1983, Section 5], where it is shown that these modules together with the trivial module form the discrete part of the classification of unitary highest weight modules with one dimensional lowest $K$-type. (Note that since $\zeta$ is orthogonal to all the roots of $\mathfrak{k}, k c \zeta$ is indeed the weight of a one-dimensional $\mathfrak{k}$-module.)

We will now describe the Wallach modules more explicitly in each of the three cases. To do this, we also review some well known structural facts.

We start with the symplectic case, $\mathfrak{g}_{0}=\mathfrak{s p}(2 n, \mathbb{R})$. In this case, both $\mathfrak{g}=$ $\mathfrak{s p}(2 n, \mathbb{C})$ and $\mathfrak{k}=\mathfrak{g l}(n, \mathbb{C})$ have rank $n$. So if $\mathfrak{t}$ is a common Cartan subalgebra of $\mathfrak{g}$ and $\mathfrak{k}$, both $\mathfrak{t}$ and its dual can be identified with $\mathbb{C}^{n}$. It is standard to choose the positive compact roots to be $e_{i}-e_{j}$ for $1 \leq i<j \leq n$, and the positive noncompact roots to be $e_{i}+e_{j}$ for $i<j$ and $2 e_{i}, i=1, \ldots, n$. If we as usual denote by $\rho$ and $\rho_{c}$ the half sums of the positive roots for $\mathfrak{g}$ respectively for $\mathfrak{k}$, and by $\rho_{n}=\rho-\rho_{c}$ 
the half sum of the noncompact positive roots, then we see

$$
\rho=(n, \ldots, 1), \quad \rho_{c}=\left(\frac{n-1}{2}, \ldots,-\frac{n-1}{2}\right), \quad \rho_{n}=\left(\frac{n+1}{2}, \ldots, \frac{n+1}{2}\right) .
$$

(The entries of $\rho_{c}$ and $\rho$ decrease by one, while those of $\rho_{n}$ are constant.)

The Weyl group $W_{K}$ consists of permutations of the variables, while $W_{G}$ also contains arbitrary sign changes of the variables. The fundamental chamber for $\mathfrak{g}$ is given by the inequalities $x_{1} \geq x_{2} \geq \cdots \geq x_{n} \geq 0$, while the fundamental chamber for $\mathfrak{k}$ is given by $x_{1} \geq x_{2} \geq \cdots \geq x_{n}$. (These are the closed fundamental chambers; the open ones are given by strict inequalities.)

The simple roots corresponding to our choice of positive roots are $e_{i}-e_{i+1}$, for $i=1, \ldots, n-1$, and $2 e_{n}$. It follows that in this case $\zeta=(1,1, \ldots, 1)$ and consequently the lowest weight of the $k$-th Wallach representation is

$$
\left(\frac{k}{2}, \frac{k}{2}, \ldots, \frac{k}{2}\right) \text {. }
$$

This is also the (only) weight of the lowest $K$-type of the $k$-th Wallach module. The infinitesimal character is obtained by subtracting $\rho$ from the lowest weight. We conjugate the result to the positive Weyl chamber for $\mathfrak{g}$ and obtain

$$
\Lambda=\left(n-\frac{k}{2}, n-1-\frac{k}{2}, \ldots, \frac{k}{2}, \frac{k}{2}-1, \frac{k}{2}-1, \frac{k}{2}-2, \frac{k}{2}-2, \ldots\right),
$$

which ends with $1,1,0$ if $k$ is even and with $\frac{1}{2}, \frac{1}{2}$ if $k$ is odd.

We will also need to describe other $K$-types of Wallach modules. An explicit description can be found for example in [Nishiyama et al. 2001, Corollary 6.3]. The result is that all $K$-types appear with multiplicity one, and their highest weights are

$$
\left(\frac{k}{2}, \frac{k}{2}, \ldots, \frac{k}{2}\right)+\left(d_{1}, d_{2}, \ldots, d_{k}, 0, \ldots, 0\right),
$$

for arbitrary even integers $d_{1} \geq \cdots \geq d_{k} \geq 0$.

The proof of this fact relies on a construction of Wallach representations via Howe duality. The relevant dual pair here is

$$
\operatorname{Sp}(2 n, \mathbb{R}) \times O(k) \subset \mathrm{Sp}(2 n k, \mathbb{R}) .
$$

This dual pair construction is in turn related to invariant theory, more specifically the first and second fundamental theorems of invariant theory, as described in [Howe 1989] and [Howe 1995, Chapters 2 and 3]. Another relevant reference is [Kashiwara and Vergne 1978]. In the following we outline the setting of this approach.

Let $\mathrm{M}_{n, k}$ be the complex vector space of $n \times k$ matrices with the linear action of the orthogonal group $O(k)$ by the right matrix multiplication. This action induces 
an action of $O(k)$ on the algebra $\mathscr{P}\left(\mathrm{M}_{n, k}\right)$ of polynomial functions on $\mathrm{M}_{n, k}$ by algebra automorphisms. Let

$$
V_{k}^{+}=\mathscr{P}\left(\mathrm{M}_{n, k}\right){ }^{O(k)}
$$

be the subalgebra of the $O(k)$-invariants in $\mathscr{P}\left(\mathrm{M}_{n, k}\right)$. Then $V_{k}^{+}$can be expressed as a quotient of the algebra $\mathscr{P}\left(S^{2}\left(\mathbb{C}^{n}\right)\right)$ of polynomial functions by the determinantal ideal of rank $k$. The symplectic Lie algebra $\mathfrak{s p}(2 n, \mathbb{R})$ acts on $\mathscr{P}\left(\mathrm{M}_{n, k}\right)$ by polynomial coefficient differential operators. This action commutes with the action of $O(k)$, and hence $V_{k}^{+}$acquires the structure of a module for $\mathfrak{s p}(2 n, \mathbb{R})$ and hence also for $\mathfrak{g}=\mathfrak{s p}(2 n, \mathbb{C})$. Furthermore, this module is the Harish-Chandra module of an irreducible unitary representation of the metaplectic double cover $\widetilde{\operatorname{Sp}}(2 n, \mathbb{R})$ of $\operatorname{Sp}(2 n, \mathbb{R}) .^{2}$ This representation of $\widetilde{\operatorname{Sp}}(2 n, \mathbb{R})$ is called the theta-lift of the trivial representation of $O(k)$. The corresponding $(\mathfrak{g}, K)$ module $V_{k}^{+}$is also referred to as the theta-lift of the trivial representation of $O(k)$.

In this setting it is not too difficult to prove that the $K$-types of $V_{k}^{+}$are indeed given by (2.2). See [Nishiyama et al. 2001, Section 6] for more details.

It will be good for our purposes to study the Wallach representation $V_{k}^{+}$together with another module $V_{k}^{-}$, which is obtained as the theta-lift of the sign representation of $O(k)$, that is, as the isotypic component of the sign representation $O(k)$ on $\mathscr{P}\left(\mathrm{M}_{n, k}\right)$. Its $K$-types are given again by (2.2), but now $d_{1} \geq \cdots \geq d_{k} \geq 1$ are arbitrary odd integers. The module $V_{k}^{-}$is another unitary lowest weight module. However its lowest $K$-type is not scalar, as it has highest weight

$$
\left(\frac{k}{2}+1, \ldots, \frac{k}{2}+1, \frac{k}{2} \ldots, \frac{k}{2}\right),
$$

with the first $k$ entries equal, and the last $n-k$ entries also equal. We will be able to find Dirac cohomology of $V_{k}^{-}$simultaneously with $V_{k}^{+}$, with no additional work. Moreover, replacing the Wallach module $V_{k}^{+}$with the module $V_{k}=V_{k}^{+} \oplus V_{k}^{-}$, will actually make our results more uniform when compared with the orthogonal and unitary case, where we do not have analogs of $V_{k}^{-}$. The module $V_{k}$ can be described as the subalgebra of the invariants of the special orthogonal group $\mathrm{SO}(k)$ acting on $\mathscr{P}\left(\mathbf{M}_{n, k}\right)$.

To conclude the discussion of the symplectic case, we mention that for $k=1$, $V_{1}^{+}$and $V_{1}^{-}$are respectively the even and odd oscillator (Weil, metaplectic) representations of $\widetilde{\mathrm{Sp}}(2 n, \mathbb{R})$.

We now consider the orthogonal case, $\mathfrak{g}_{0}=\mathfrak{s o}^{*}(2 n, \mathbb{R})$. The Lie algebras $\mathfrak{g}=$ $\mathfrak{s o}(2 n, \mathbb{C})$ and $\mathfrak{k}=\mathfrak{g l}(n, \mathbb{C})$ both have rank $n$, and we choose a common Cartan subalgebra $t$ in both of them. Both $\mathfrak{t}$ and $\mathfrak{t}^{*}$ are identified with $\mathbb{C}^{n}$. We choose the

${ }^{2}$ In particular, this means that the relevant $K$ here is not $U(n)$ which is the maximal compact subgroup of $\operatorname{Sp}(2 n, \mathbb{R})$, but the double cover of $U(n)$ which is the maximal compact subgroup of $\widetilde{\mathrm{Sp}}(2 n, \mathbb{R})$. This is reflected by the possible presence of half integers in the expression (2.2). 
positive compact roots to be $e_{i}-e_{j}$ for $1 \leq i<j \leq n$, and the noncompact positive roots to be $e_{i}+e_{j}$ for $1 \leq i<j \leq n$. Thus

$$
\rho=(n-1, \ldots, 0), \quad \rho_{c}=\left(\frac{n-1}{2}, \ldots,-\frac{n-1}{2}\right), \quad \rho_{n}=\left(\frac{n-1}{2}, \ldots, \frac{n-1}{2}\right) .
$$

(The entries of $\rho_{c}$ and $\rho$ decrease by one, while those of $\rho_{n}$ are constant.)

The Weyl group $W_{K}$ consists of permutations of the variables, while $W_{G}$ also contains arbitrary sign changes of even number of the variables. The fundamental chamber for $\mathfrak{g}$ is given by the inequalities $x_{1} \geq x_{2} \geq \cdots \geq x_{n-1} \geq\left|x_{n}\right|$, while the fundamental chamber for $\mathfrak{k}$ is given by $x_{1} \geq x_{2} \geq \cdots \geq x_{n}$. (These are the closed fundamental chambers; the open ones are given by strict inequalities.)

The simple roots corresponding to our choice of positive roots are $e_{i}-e_{i+1}$, for $i=1, \ldots, n-1$, and $e_{n-1}+e_{n}$. It follows that in this case $\zeta=\left(\frac{1}{2}, \frac{1}{2}, \ldots, \frac{1}{2}\right)$, so the lowest weight of the $k$-th Wallach representation $V_{k}, k=1,2, \ldots,\left[\frac{n}{2}\right]$, is

$$
(k, k, \ldots, k) \text {. }
$$

This is also the (only) weight of the lowest $K$-type of $V_{k}$. The infinitesimal character is obtained by subtracting $\rho$ from the lowest weight. We conjugate the result to the positive Weyl chamber for $\mathfrak{g}$ and obtain

$$
(n-k-1, n-k-2, \ldots, k+1, k, k, k-1, k-1, \ldots, 1,1,0) .
$$

Note that $n-k-1 \geq k+1$, so there is at least one nonrepeated entry before $k, k$. All $K$-types of $V_{k}$ are of multiplicity one, and their highest weights are

$$
(k, \ldots, k)+\left(d_{1}, d_{1}, d_{2}, d_{2}, \ldots, d_{k}, d_{k}, 0, \ldots, 0\right),
$$

for arbitrary integers $d_{1} \geq \cdots \geq d_{k} \geq 0$. See, for example, Corollary 6.9 of [Nishiyama et al. 2001]. This follows from the fact that each $V_{k}$ is the thetalift of the trivial representation of the compact factor $\operatorname{Sp}(2 k)$ of the dual pair $\mathrm{SO}^{*}(2 n, \mathbb{R}) \times \mathrm{Sp}(2 k) \subset \mathrm{Sp}(4 n k, \mathbb{R})$. Each $V_{k}$ is the Harish-Chandra module of a unitary representation of $\mathrm{SO}^{*}(2 n, \mathbb{R})$.

Finally, let us consider the case $\mathfrak{g}_{0}=\mathfrak{u}(p, q)$. It is slightly more convenient to work with $\mathfrak{g}_{0}=\mathfrak{u}(p, q)$, which leads easily to the desired conclusion for $\mathfrak{g}_{0}=$ $\mathfrak{s u}(p, q)$.

The Lie algebras

$$
\begin{aligned}
& \mathfrak{g}=\mathfrak{u}(p, q)_{\mathbb{C}}=\mathfrak{g l}(p+q, \mathbb{C}), \\
& \mathfrak{k}=(\mathfrak{u}(p) \times \mathfrak{u}(q))_{\mathbb{C}}=\mathfrak{g l}(p, \mathbb{C}) \times \mathfrak{g l}(q, \mathbb{C})
\end{aligned}
$$

are both of rank $n=p+q$. Let $\mathfrak{t}$ be a Cartan subalgebra of both $\mathfrak{g}$ and $\mathfrak{k}$. Then both $\mathfrak{t}$ and $\mathfrak{t}^{*}$ can be identified with $\mathbb{C}^{n}$. We choose the positive roots for $\mathfrak{t}$ in $\mathfrak{g}$ to be $e_{i}-e_{j}, 1 \leq i<j \leq n$. Among them, the compact ones are those for which 
either $1 \leq i<j \leq p$ or $p+1 \leq i<j \leq n$, while the noncompact ones are those for which $1 \leq i \leq p$ and $p+1 \leq j \leq n$. Thus

$$
\begin{aligned}
\rho & =\frac{1}{2}(p+q-1, p+q-3, \ldots,-(p+q-1)), \\
\rho_{c} & =\frac{1}{2}(p-1, p-3, \ldots,-(p-1) \mid q-1, q-3, \ldots,-(q-1)), \\
\rho_{n} & =\frac{1}{2}(q, \ldots, q \mid-p, \ldots,-p) .
\end{aligned}
$$

(We will often separate the first $p$ coordinates from the last $q$ coordinates by a bar).

The Weyl group $W_{G}$ consists of all permutations of $n=p+q$ elements, while $W_{K}$ consists of those permutations that permute separately the first $p$ elements and the last $q$ elements. The closed fundamental chamber for $\mathfrak{g}$ consists of all $\left(x_{1}, \ldots, x_{p+q}\right)$ such that $x_{1} \geq \cdots \geq x_{p+q}$, while the closed fundamental chamber for $\mathfrak{q}$ consists of all $\left(x_{1} \ldots, x_{p} \mid y_{1}, \ldots, y_{q}\right)$ such that $x_{1} \geq \cdots \geq x_{p}$ and $y_{1} \geq \cdots \geq y_{q}$.

To describe the Wallach representations, we first note that the simple roots corresponding to our choice of positive roots are $e_{i}-e_{i+1}, i=1, \ldots, n-1$. All of these are compact except for $e_{p}-e_{p+1}$. It follows that $\zeta=(a, \ldots, a \mid b, \ldots, b)$ for some $a$ and $b$ such that $a-b=1$. For $\mathfrak{g}_{0}=\mathfrak{s u}(p, q)$ it would also be required that $p a+q b=0$, so it would follow that $a=p / n$ and $b=-q / n$. The lowest weights of the Wallach modules $V_{k}, k=1,2, \ldots, \min (p, q)-1$, would then be

$$
\left(\frac{k p}{n}, \ldots, \frac{k p}{n} \mid-\frac{k q}{n}, \ldots,-\frac{k q}{n}\right) .
$$

For $\mathfrak{g}_{0}=\mathfrak{u}(p, q)$ we can however simplify the lowest weight by twisting the module by central character $(k(p-q)) / 2 n(1, \ldots, 1)$, and thus work with the lowest weight of the form

$$
\left(\frac{k}{2}, \ldots, \frac{k}{2} \mid-\frac{k}{2}, \ldots,-\frac{k}{2}\right) \text {. }
$$

This twisting of course does not change the modules very much, and we will call the twisted modules the Wallach modules for $\mathfrak{u}(p, q)$ and denote them by $V_{k}, k=1,2, \ldots, \min (p, q)-1$.

To obtain the infinitesimal character of $V_{k}$, we as usual subtract $\rho$ from the lowest weight. This has the following $\mathfrak{g}$-dominant representative:

$$
\begin{aligned}
\Lambda=\frac{1}{2}( & p+q-1-k, p+q-3-k, \ldots, p-q+1+k, \\
& p-q-1+k, p-q-1+k, \ldots, p-q+1-k, p-q+1-k, \\
& p-q-1-k, p-q-3-k, \ldots,-p-q+1+k) .
\end{aligned}
$$

Here after multiplying by $\frac{1}{2}$ the first row consists of $q-k$ coordinates decreasing by $\frac{1}{2} \cdot 2=1$, the second row consists of $k$ pairs of equal coordinates with the value of each pair being 1 less than the value of the previous pair, and the third row consists of $p-k$ coordinates decreasing by 1 . 
All $K$-types of $V_{k}$ are of multiplicity one, and their highest weights are

$$
\left(\frac{k}{2}, \ldots, \frac{k}{2} \mid-\frac{k}{2}, \ldots,-\frac{k}{2}\right)+\left(d_{1}, \ldots, d_{k}, 0, \ldots, 0 \mid 0, \ldots, 0,-d_{k}, \ldots,-d_{1}\right),
$$

for arbitrary integers $d_{1} \geq \cdots \geq d_{k} \geq 0$. See for example Corollary 6.7 of [Nishiyama et al. 2001]. This follows from the fact that each $V_{k}$ is the theta-lift of the trivial representation of the compact factor $U(k)$ of the dual pair

$$
U(p, q) \times U(k) \subset \operatorname{Sp}(2(p+q) k, \mathbb{R}) .
$$

Each $V_{k}$ is the Harish-Chandra module of a unitary representation of the double cover of $U(p, q)$ defined as the preimage of $U(p, q)$ in the metaplectic double cover of $\operatorname{Sp}(2(p+q) k, \mathbb{R})$.

\section{Preliminaries on Dirac cohomology}

In this section we review basic facts about Dirac cohomology with emphasis on the special Hermitian setting we are considering in this paper. For more details, see [Huang and Pandžić 2006, Chapter 3].

Let $G$ be a connected real reductive Lie group with Cartan involution $\Theta$ and assume that $K=G^{\Theta}$ is a maximal compact subgroup of $G$. Let $\mathfrak{g}=\mathfrak{k} \oplus \mathfrak{p}$ be the Cartan decomposition of the complexified Lie algebra of $G$ corresponding to $\Theta$. We fix a nondegenerate invariant symmetric bilinear form $B$ on $\mathfrak{g}$, equal to the Killing form on the semisimple part of $\mathfrak{g}$.

Let $U(\mathfrak{g})$ be the universal enveloping algebra of $\mathfrak{g}$ and let $C(\mathfrak{p})$ be the Clifford algebra of $\mathfrak{p}$ with respect to $B$. The Dirac operator $D \in U(\mathfrak{g}) \otimes C(\mathfrak{p})$ is defined as

$$
D=\sum_{i} b_{i} \otimes d_{i}
$$

where $b_{i}$ is a basis of $\mathfrak{p}$, and $d_{i}$ is the dual basis with respect to $B$. It is easy to check that $D$ does not depend on the choice of the basis $b_{i}$ and moreover it is $K$-invariant for the diagonal action of $K$ given by adjoint actions on both factors.

In the Hermitian setting, the $\mathfrak{k}$-module $\mathfrak{p}$ decomposes as $\mathfrak{p}^{+} \oplus \mathfrak{p}^{-}$, where $\mathfrak{p}^{ \pm}$are isomorphic as modules for the semisimple part of $\mathfrak{k}$, while the center of $\mathfrak{k}$ acts on them by dual characters. Let $m=\operatorname{dim} \mathfrak{p}^{+}=\operatorname{dim} \mathfrak{p}^{-}$. We now choose the basis $b_{i}$ in the following way. Let $\Delta(\mathfrak{g})$ be the set of roots of the compact Cartan subalgebra $t$ in $\mathfrak{g}$. Let $\Delta^{+}(\mathfrak{g})$ denote a fixed choice of positive roots which is compatible with $\mathfrak{p}^{+}$, that is, the noncompact positive roots are exactly the $\mathfrak{t}$-weights $\alpha_{1}, \ldots, \alpha_{m}$ of $\mathfrak{p}^{+}$. For each $\alpha_{i}$ we choose a root vector $e_{i}$. Let $f_{i}$ be the root vector for the root $-\alpha_{i}$ such that $B\left(e_{i}, f_{i}\right)=1$. Then for the basis $b_{i}$ of $\mathfrak{p}$ we choose $e_{1}, \ldots e_{m} ; f_{1}, \ldots f_{m}$. 
The dual basis is then $f_{1}, \ldots f_{m} ; e_{1}, \ldots e_{m}$, and hence

$$
D=\sum_{i=1}^{m} e_{i} \otimes f_{i}+f_{i} \otimes e_{i}
$$

Let $X$ be a $(\mathfrak{g}, K)$-module. To get a module for the algebra $U(\mathfrak{g}) \otimes C(\mathfrak{p})$, we tensor $X$ with an irreducible (spin) module $S$ for $C(\mathfrak{p})$. In the Hermitian case, $\mathfrak{p}$ is even-dimensional, hence there is only one irreducible $C(\mathfrak{p})$-module up to isomorphism, and it can be constructed as $S=\bigwedge \mathfrak{p}^{+}$.

Now $X \otimes S$ is a $(U(\mathfrak{g}) \otimes C(\mathfrak{p}), \tilde{K})$ module, where $\tilde{K}$ is the spin double cover of $K$, that is, the pullback of the double cover $\operatorname{Spin}\left(\mathfrak{p}_{0}\right) \rightarrow \mathrm{SO}\left(\mathfrak{p}_{0}\right)$ by the action map $K \rightarrow \mathrm{SO}\left(\mathfrak{p}_{0}\right)$. The action of $U(\mathfrak{g}) \otimes C(\mathfrak{p})$ on $X \otimes S$ is the obvious one, and $\tilde{K}$ acts on both factors, on $X$ through $K$ and on $S$ through the spin group $\operatorname{Spin}\left(\mathfrak{p}_{0}\right) \subset C(\mathfrak{p})$. The copy of $\mathfrak{k}$ in $U(\mathfrak{g}) \otimes C(\mathfrak{p})$ corresponding to $\tilde{K}$ is

$$
\mathfrak{k}_{\Delta}=\{Y \otimes 1+1 \otimes \alpha(Y) \mid Y \in \mathfrak{k}\} .
$$

Here $\alpha$ is the complexification of the map $\mathfrak{k}_{0} \rightarrow \mathfrak{s o}\left(\mathfrak{p}_{0}\right) \cong \bigwedge^{2} \mathfrak{p}_{0} \hookrightarrow C\left(\mathfrak{p}_{0}\right)$ given by the adjoint action followed by the skew symmetrization.

Now the Dirac operator $D$ acts on $X \otimes S$, and the Dirac cohomology of $X$ is the $\tilde{K}$-module

$$
H_{D}(X)=\operatorname{Ker} D /(\operatorname{Im} D \cap \operatorname{Ker} D) .
$$

If $X$ is unitary, then it is well known that $D$ is self-adjoint with respect to the inner product on $X \otimes S$ induced by the invariant inner product on $X$ and the usual inner product on $S$ (see [Wallach 1988, p. 367] or [Huang and Pandžić 2006, p. 63].) It follows that $\operatorname{Ker} D \cap \operatorname{Im} D=0$, and hence

$$
H_{D}(X)=\operatorname{Ker} D=\operatorname{Ker} D^{2} .
$$

We will now summarize some earlier results. We denote by $E_{\gamma}$ the irreducible $\tilde{K}$-module with highest weight $\gamma \in \mathfrak{t}^{*}$.

Theorem 3.1 [Huang and Pandžić 2002]. Let $X$ be an irreducible unitary $(\mathfrak{g}, K)$ module with infinitesimal character $\Lambda \in \mathfrak{t}^{*}$. Assume that the $\tilde{K}$-module $E_{\gamma}$ appears in $X \otimes S$. Then the following are equivalent:

(1) the $\gamma$-isotypic component of $X \otimes S$ is contained in $H_{D}(X)$;

(2) $\Lambda$ is conjugate to $\gamma+\rho_{c}$ under the Weyl group $W_{G}=W(\mathfrak{g}, \mathfrak{t})$;

(3) $\|\Lambda\|=\left\|\gamma+\rho_{c}\right\|$.

The implication $(1) \Rightarrow(2)$ is true without the unitarity assumption. This is one of the main results of [Huang and Pandžić 2002], conjectured by Vogan. The implication (2) $\Rightarrow(3)$ is obvious. Let us briefly recall why (3) implies (1). By 
[Parthasarathy 1972] (see [Huang and Pandžić 2006, Proposition 3.1.6]),

$$
D^{2}=-\left(\Omega_{\mathfrak{g}}+\|\rho\|^{2}\right)+\left(\Omega_{\mathfrak{k}_{\Delta}}+\left\|\rho_{c}\right\|^{2}\right) .
$$

Here $\Omega_{\mathfrak{g}}$ is the Casimir element of $U(\mathfrak{g})$ and $\Omega_{\mathfrak{k}_{\Delta}}$ is the Casimir element of $U\left(\mathfrak{k}_{\Delta}\right)$. Since $\Omega_{\mathfrak{g}}$ acts on $X$ by the scalar $\|\Lambda\|^{2}-\|\rho\|^{2}$ and $\Omega_{\mathfrak{k}_{\Delta}}$ acts on a $\tilde{K}$-type $E_{\gamma}$ by the scalar $\left\|\gamma+\rho_{c}\right\|^{2}-\left\|\rho_{c}\right\|^{2}$, we see that $D^{2}=0$ on the isotypic component $(X \otimes S)(\gamma)$ if and only if $\|\Lambda\|=\left\|\gamma+\rho_{c}\right\|$.

Another useful consequence of the fact that $D$ is self-adjoint for unitary $X$, and hence $D^{2} \geq 0$, is Parthasarathy's Dirac inequality:

Proposition 3.3 [Parthasarathy 1980, Lemma 2.5]. Let $X$ be a unitary $(\mathfrak{g}, K)$ module with infinitesimal character $\Lambda \in \mathfrak{t}^{*}$. Let $E_{\gamma}$ be any $\tilde{K}$-type contained in $X \otimes S$. Then

$$
\|\Lambda\| \leq\left\|\gamma+\rho_{c}\right\| .
$$

This simply follows from writing out the inequality $D^{2} \geq 0$ on the isotypic component $(X \otimes S)(\gamma)$, using (3.2).

The following remark will be useful for our calculations.

Proposition 3.4. Let $X$ be an irreducible unitary $(\mathfrak{g}, K)$-module with infinitesimal character $\Lambda$. Assume that $H_{D}(X)$ contains a $\tilde{K}$-type

$$
E_{\gamma} \subset E_{\mu} \otimes E_{\sigma} \subset X \otimes S,
$$

where $E_{\mu} \subset X$ and $E_{\sigma} \subset S$. Then $E_{\gamma}$ is the PRV component of $E_{\mu} \otimes E_{\sigma}$, that is, $\gamma$ is conjugate to $\sigma$ plus the lowest weight of $E_{\mu}$ under the Weyl group $W_{K}$ of $\mathfrak{k}$.

Proof. By Proposition 3.3, for any $E_{\gamma^{\prime}} \subset X \otimes S$, we have

$$
\left\|\gamma^{\prime}+\rho_{c}\right\| \geq\|\Lambda\| \text {. }
$$

Moreover, since $D^{2}=\left\|\gamma^{\prime}+\rho_{c}\right\|^{2}-\|\Lambda\|^{2}$ on $E_{\gamma^{\prime}}$, and $D^{2} \geq 0$, the expression $\left\|\gamma^{\prime}+\rho_{c}\right\|$ is the smallest possible when $\gamma^{\prime}=\gamma$. In particular, this is true for all $\gamma^{\prime}$ such that $E_{\gamma^{\prime}}$ is contained in $E_{\mu} \otimes E_{\sigma}$, which means that $E_{\gamma}$ is the PRV component of $E_{\mu} \otimes E_{\sigma}$ [Parthasarathy et al. 1967; Wallach 1988, 9.1.6].

We can now describe the setting for calculating Dirac cohomology of an irreducible unitary $(\mathfrak{g}, K)$ module $X$ more precisely. We are looking for $\tilde{K}$-types $E_{\gamma}$ in $X \otimes S$ such that $\gamma+\rho_{c}$ is conjugate to the infinitesimal character $\Lambda$ of $X$ by some $w \in W_{G}$. We can assume that $\Lambda$ is $\mathfrak{g}$-dominant. The following obvious fact will be useful.

Proposition 3.5. The only possible $\tilde{K}$-types in $H_{D}(X)$ are of the form $E_{w \Lambda-\rho_{c}}$, with $w \in W_{G}$ such that $w \Lambda$ is dominant regular for $\mathfrak{k}$. In particular, $w$ is in $W^{1}$, where $W^{1} \subset W_{G}$ consists of those $w$ that take the fundamental Weyl chamber for $\mathfrak{g}$ into the fundamental Weyl chamber for $\mathfrak{k}$. 
Each coset of $W_{K}$ in $W_{G}$ contains exactly one element of $W^{1}$. In particular, $W^{1}$ has $\left|W_{G}\right| /\left|W_{K}\right|$ elements.

On the other hand, it is well known (see [Wallach 1988] or [Huang and Pandžić 2006, Section 2.3], for instance) that the spin module $S$ for $\mathfrak{k}$ decomposes as

$$
S=\bigoplus_{\sigma \in W^{1}} E_{\sigma \rho-\rho_{c}} .
$$

Thus we see that for a $K$-type $E_{\mu}$ of $X$ and a $\tilde{K}$-type $E_{\sigma \rho-\rho_{c}}$ of $S$, the PRV component of $E_{\mu} \otimes E_{\sigma \rho-\rho_{c}}$ will contribute to $H_{D}(X)$ if and only if

$$
w \Lambda-\rho_{c}=\left(\sigma \rho-\rho_{c}+\mu^{-}\right)^{\prime} .
$$

Here $\mu^{-}$denotes the lowest weight of $E_{\mu}$, and $\left(\sigma \rho-\rho_{c}+\mu^{-}\right)^{\prime}$ denotes the $\mathfrak{k}^{-}$ dominant $W_{K}$-conjugate of $\sigma \rho-\rho_{c}+\mu^{-}$. This will be the starting point for our calculations. It will turn out that the calculation is slightly simpler if we add $\rho_{n}$ to both sides of the equation:

$$
w \Lambda-\rho_{c}+\rho_{n}=\left(\sigma \rho-\rho_{c}+\rho_{n}+\mu^{-}\right)^{\prime} .
$$

Note that since $\rho_{n}$ is $W_{K}$-invariant, it is legitimate to put it inside the parentheses on the right side.

To end this section, let us note that for unitary lowest weight modules the Dirac cohomology never vanishes.

Proposition 3.7. Let $X$ be an irreducible unitary lowest weight $(\mathfrak{g}, K)$-module with lowest $K$-type $E_{\mu}$. Then $E_{\mu} \otimes \mathbb{C} \cdot 1 \subset X \otimes S$ is contained in $H_{D}(X)$. In particular, $H_{D}(X)$ is not zero.

Proof. This is straightforward: both $E_{\mu} \subset X$ and $\mathbb{C} \cdot 1 \subset S$ are annihilated by all $f_{i} \in \mathfrak{p}^{-}$. Thus every term of $D=\sum e_{i} \otimes f_{i}+f_{i} \otimes e_{i}$ kills $E_{\mu} \otimes \mathbb{C} \cdot 1$.

\section{The case of $\mathfrak{s p}(2 n, \mathbb{R})$}

We are going to use the facts about the structure of the pair $(\mathfrak{g}, \mathfrak{k})$ which we reviewed in Section 2. Besides that, we also need to describe the subset $W^{1} \subset W_{G}$ consisting of those $w \in W_{G}$ which conjugate the fundamental chamber for $\mathfrak{g}$ into the fundamental chamber for $\mathfrak{k}$. Alternatively, $W^{1}$ can be described as the set of the minimal length representatives of the left $W_{K}$-cosets in $W_{G}$.

In the symplectic case, $W^{1}$ may be parametrized by $\mathbb{Z}_{2}^{n}$. Namely, for any choice of sign changes $\epsilon=\left(\epsilon_{1}, \ldots, \epsilon_{n}\right)$, there is a unique permutation $\tau$ of the variables such that for any $\mathfrak{g}$-dominant $\left(x_{1}, \ldots x_{n}\right), \tau\left(\epsilon_{1} x_{1}, \ldots, \epsilon_{n} x_{n}\right)$ is $\mathfrak{k}$-dominant. We will be slightly imprecise and identify $\epsilon$ with the corresponding element of $W^{1}$.

Recall that the modules we are interested in are $V_{k}=V_{k}^{+} \oplus V_{k}^{-}$, where $k$ in an 
integer such that $1 \leq k \leq n-1$. The infinitesimal character of $V_{k}$ was given in (2.1). We now rewrite it as

$$
\Lambda=\left(\frac{k}{2}+n-k, \frac{k}{2}+n-k-1, \ldots, \frac{k}{2}, \frac{k}{2}-1, \frac{k}{2}-1, \frac{k}{2}-2, \frac{k}{2}-2, \ldots\right)
$$

ending in the same way as before, by $1,1,0$ if $k$ is even and by $\frac{1}{2}, \frac{1}{2}$ if $k$ is odd.

By Proposition 3.5, any $w$ involved in (3.6) must put a minus on exactly one member of each pair of repeated coordinates. It does not matter which of the two gets a minus, since $w \Lambda$ will be the same in each case. In other words, we see that

$$
w=\epsilon_{1} \epsilon_{2} \ldots \epsilon_{n-k+1}( \pm \mp)( \pm \mp) \ldots( \pm \mp)( \pm),
$$

where each of the pairs in parentheses can be $(+-)$ or $(-+)$, while the last sign $( \pm)$ appears when $k$ is even. All such choices give the same $w \Lambda$, which is thus determined by the sequence $\epsilon_{1} \epsilon_{2} \ldots \epsilon_{n-k+1}$. We will therefore work only with $w$ of the form

$$
w=\epsilon_{1} \epsilon_{2} \ldots \epsilon_{n-k+1}(+-)(+-) \ldots(+-)(+) .
$$

This means that of all possible $w$ defining the same weight $w \Lambda$ we choose the shortest one. Namely, each time when we have $(-+)$ instead of $(+-)$, it requires an additional transposition to keep $\mathfrak{g}$-dominant weights $\mathfrak{k}$-dominant.

Theorem 4.3. The Dirac cohomology of $V_{k}$ is

$$
H_{D}\left(V_{k}\right)=\bigoplus_{w} E_{w \Lambda-\rho_{c}}
$$

with the summation over all $w$ as in (4.2). All ingredients of the formula (3.6) are uniquely determined by $w$ : $\sigma$ is given by

$$
\sigma=--\cdots-\epsilon_{1} \epsilon_{2} \ldots \epsilon_{n-k}
$$

and the corresponding $K$-type in $V_{k}$ is the one with highest weight of the form (2.2) given by

$$
d_{1}=\cdots=d_{k}=\text { the number of pluses in the sequence } \epsilon=\left(\epsilon_{1}, \ldots, \epsilon_{n-k+1}\right) .
$$

In particular, $H_{D}\left(V_{k}^{+}\right)$contains all $E_{w \Lambda-\rho_{c}}$ with $w$ such that the number of pluses in the sequence $\epsilon$ is even, while $H_{D}\left(V_{k}^{-}\right)$contains all $E_{w \Lambda-\rho_{c}}$ with $w$ such that the number of pluses in the sequence $\epsilon$ is odd.

Proof. By (2.2), the lowest weights of the $K$-types of $V_{k}$ appearing in the right side of (3.6) are

$$
\left(0, \ldots, 0, d_{k}, \ldots, d_{1}\right)+\left(\frac{k}{2}, \ldots, \frac{k}{2}\right)
$$


Since $(k / 2, \ldots, k / 2)$ is invariant under $W_{K}$, we can subtract it from both sides of (3.6). Now we can write out the new left side of (3.6) using the expression (4.1) for $\Lambda$ and (4.2) for $w$. To do this, let

$$
i_{1}>i_{2}>\cdots>i_{r} \quad \text { and } \quad j_{1}<j_{2}<\cdots<j_{s}
$$

be the integers in $[0, n-k]$ such that $\epsilon_{n-k+1-i_{t}}=+, t=1, \ldots, r$, respectively $\epsilon_{n-k+1-j_{u}}=-, u=1, \ldots, s$. This means that $r+s=n-k+1$, and that

$$
\left\{i_{1}, \ldots, i_{r}, j_{1}, \ldots, j_{s}\right\}=\{0,1, \ldots, n-k\} .
$$

Note that the sequence $\epsilon_{1}, \ldots, \epsilon_{n-k+1}$ is completely determined by $i$ s and $j \mathrm{~s}$ and vice versa.

Now we have

$$
\begin{aligned}
w \Lambda-\rho_{c} & +\rho_{n}-\left(\frac{k}{2}, \ldots, \frac{k}{2}\right) \\
& =\left(i_{1}+1, \ldots, i_{r}+r, r, \ldots, r, r-j_{1}, r+1-j_{2}, \ldots, n-k-j_{s}\right) .
\end{aligned}
$$

Note that $r$ appears in $k-1$ places, from the $(r+1)$-st place to the $(r+k-1)$-st.

On the other hand, the right side of (3.6) after subtracting $(k / 2, \ldots, k / 2)$ becomes

$$
\left(\sigma \rho-\rho_{c}+\rho_{n}+\left(0, \ldots, 0, d_{k}, \ldots, d_{1}\right)\right)^{\prime} .
$$

(Recall that the prime means taking the $\mathfrak{k}$-dominant $W_{K}$-conjugate.)

Since the largest component of (4.4) is $i_{1}+1 \leq n-k+1$, we see that for (3.6) to hold, $\sigma \rho$ cannot have entries $n, n-1, \ldots, n-k+1$. Thus the starting $k$ signs of $\sigma$ are all minuses, that is,

$$
\sigma=-\cdots-\delta_{1} \ldots \delta_{n-k} .
$$

Using similar notation as before, we see that

$$
\sigma \rho=\left(I_{1}, \ldots, I_{a},-J_{1}, \ldots,-J_{b},-(n-k+1),-(n-k+2), \ldots,-n\right),
$$

where $I_{1}>\cdots>I_{a}$ and $J_{1}<\cdots<J_{b}$ are integers in $[1, n-k]$ determined by $\delta_{1}, \ldots, \delta_{n-k}$. In particular, $a+b=n-k$,

$$
\left\{I_{1}, \ldots, I_{a}, J_{1}, \ldots, J_{b}\right\}=\{1, \ldots, n-k\}
$$

and $I \mathrm{~s}$ and $J \mathrm{~s}$ correspond to $\delta \mathrm{s}$ via

$$
\delta_{n-k+1-I_{u}}=+, \quad \delta_{n-k+1-J_{v}}=-,
$$

for all indices $u$ and $v$.

It is now easily seen that (4.5) equals

$$
\left(I_{1}+1, \ldots, I_{a}+a,-J_{1}+a+1, \ldots,-J_{b}+a+b, d_{k}, \ldots, d_{1}\right)^{\prime} .
$$


Thus $w \Lambda-\rho_{c}$ contributes to $H_{D}\left(V_{k}\right)$ if and only if (4.4) equals (4.7). Clearly, this is the case if $I \mathrm{~s}$ are equal to $i \mathrm{~s}, J \mathrm{~s}$ are equal to $j \mathrm{~s}$, and all $d \mathrm{~s}$ are equal to $r$. In this case, $\delta_{i}=\epsilon_{i}$ for $i=1, \ldots, n-k$. Thus to prove the theorem, we must show that this is the only possibility.

Either $i_{r}=0$ and $j_{1}>0$, or $i_{r}>0$ and $j_{1}=0$. The proof in each of these two cases is analogous, so we will assume that $i_{r}=0$ and $j_{1}>0$. Thus (4.4) is

$$
\left(i_{1}+1, \ldots, i_{r-1}+r-1, r, \ldots, r, r-j_{1}, \ldots, n-k-j_{s}\right),
$$

where the $k$ entries from $r$-th to $(r-1+k)$-th are equal to $r$, entries before these $k$ are $\geq r$, and entries after the $k r$ s are $<r$.

We claim that $a=r-1$. To see this, assume first that $a<r-1$. Since only the first $a$ or the last $k$ entries of (4.7) can be $\geq a+1$, and since $r>a+1$, we see that the number of entries of (4.7) that are $\geq r$ is at most $a+k$. On the other hand, the number of entries of (4.8) that are $\geq r$ is $r-1+k>a+k$. Hence (4.7) cannot be equal to (4.8) or (4.4) in this case.

Similarly, if $a>r-1$, then the first $a \geq r$ entries of (4.7) are $\geq a+1>r$. On the other hand, the $r$-th entry of (4.8) is $r$.

So indeed $a=r-1$. Now we see that the first $a$ terms or (4.7) are $\geq a+1=r$, and the next $b$ terms are all $<a+1=r$. On the other hand, (4.8) has $r-1+k$ entries $\geq r$. So we conclude that for (4.7) to be equal to (4.8), $d_{1}, \ldots, d_{k}$ must all be equal to $r$. Hence also $I \mathrm{~s}$ must be equal to $i$ s and $J \mathrm{~s}$ must be equal to $j \mathrm{~s}$, as claimed.

Theorem 4.3 explicitly exhibits the pairs $(\mu, \bar{\sigma}) \in \mathfrak{t}^{*} \times \mathfrak{t}^{*}$, such that $E_{\mu}$ is a $K$-type of the module $X=V_{k}$ and $E_{\bar{\sigma}}$ is a $\tilde{K}$-type of the spin module $S$, and such that the PRV component of $E_{\mu} \otimes E_{\bar{\sigma}}$ contributes to the Dirac cohomology of $X$. (Recall that both $V_{k}$ and the spin module are multiplicity free as $\mathfrak{k}$-modules.)

We now make this even more explicit by exhibiting the highest weight vectors of $E_{\mu}$ and $E_{\bar{\sigma}}$, and also the lowest weight vectors of $E_{\mu}$. These are known by [Howe 1995]. Recall that $\mathfrak{p}^{+} \cong S^{2} \mathbb{C}^{n}$, the space of $n \times n$ symmetric matrices, and that up to a twist by the character $\operatorname{det}_{n}^{k / 2}, V_{k}$ can be identified as a $K$-module with the quotient of the symmetric algebra of $\mathfrak{p}^{+}$by the $k$-th symmetric determinantal ideal, generated by minors of order $k+1$. For $1 \leq i \leq k$, let $\delta_{i}$ and $\delta_{i}^{\prime}$ be the $i \times i$ upper left and lower right corner minors of the symmetric matrix with generators in $\mathfrak{p}^{+}$.

Proposition 4.9. Let $d_{1} \geq \ldots \geq d_{k} \geq 0, d_{k+1}=0$. The element

$$
\delta_{1}^{d_{1}-d_{2}} \ldots \delta_{k}^{d_{k}-d_{k+1}} \in S\left(\mathfrak{p}^{+}\right)
$$

has $\mathfrak{k}$-highest weight $\left(2 d_{1}, \ldots, 2 d_{k}, 0, \ldots, 0\right)$ and a nonzero image in the $k$-th determinantal quotient. 
Conversely, every highest weight vector in the $k$-th determinantal quotient of $S\left(\mathfrak{p}^{+}\right)$has this form. The corresponding lowest weight vectors are obtained by replacing each $\delta_{i}$ with $\delta_{i}^{\prime}$.

Up to another twist by a character of $\mathfrak{k}=\mathfrak{g l}(n, \mathbb{C})$, the spin module is isomorphic to the exterior algebra $\Lambda\left(\mathfrak{p}^{+}\right)$. A weight basis of $\mathfrak{p}^{+}$gives rise to the basis of the exterior algebra consisting of decomposable skew-symmetric tensors. It turns out that the highest weight vectors are decomposable. Let us introduce the following partial order on the set of pairs $A=\{(i, j): 1 \leq i \leq j \leq n\}$ :

$$
(i, j) \leq(l, m) \Longleftrightarrow i \leq l \text { and } j \leq m .
$$

Proposition 4.10. For any lower-closed subset of $A$, the exterior product of the corresponding elements of $\mathfrak{p}^{+}$is a highest weight vector of $\Lambda\left(\mathfrak{p}^{+}\right)$. Conversely, every highest weight vector is proportional to the exterior product of the elements of a weight basis of $\mathfrak{p}^{+}$corresponding to a lower-closed subset of A.

The set $A$ is a lattice triangle, the upper half of the lattice $n \times n$ square $1 \leq i, j \leq n$. If $A$ is represented graphically by dots in the lattice, the lower-closed subsets of $A$ are formed by subsets that are closed under leftward and downward "slides". Each such subset is uniquely determined by its lattice boundary, consisting of alternating horizontal and vertical segments connecting the vertical axis $i=0$ with the bisector $i=j$.

To end this section, we note that the modules we are considering include the even and odd oscillator representations; thus we recover the results of [Adams 1994].

\section{The case of $\mathfrak{s} \mathfrak{o}^{*}(2 n, \mathbb{R})$}

We are going to use the facts about the structure of the pair $(\mathfrak{g}, \mathfrak{k})$ which we reviewed in Section 2. Besides that, we also need to describe the subset $W^{1} \subset W_{G}$. In the orthogonal case, $W^{1} \subset W_{G}$ may be parametrized by $\mathbb{Z}_{2}^{n-1}$. Namely, for any choice of even number of sign changes $\epsilon=\left(\epsilon_{1}, \ldots, \epsilon_{n}\right)$, there is a unique permutation $\tau$ of the variables such that for any $\mathfrak{g}$-dominant $\left(x_{1}, \ldots x_{n}\right), \tau\left(\epsilon_{1} x_{1}, \ldots, \epsilon_{n} x_{n}\right)$ is $\mathfrak{k}$-dominant. We will be slightly imprecise and identify $\epsilon$ with the corresponding element of $W^{1}$.

Recall that the modules we are interested in are $V_{k}$, where $1 \leq k \leq[n / 2]-1$ is an integer and the lowest weight of $V_{k}$ is $(k, k, \ldots, k)$.

The infinitesimal character $\Lambda$ of $V_{k}$ was given in (2.3). By Proposition 3.5, any $w$ involved in (3.6) must put a minus on exactly one member of each pair of repeated coordinates of $\Lambda$. It does not matter which of the two gets a minus, since $w \Lambda$ will be the same in each case. In other words, we see that

$$
w=\epsilon_{1} \epsilon_{2} \ldots \epsilon_{n-2 k-1}( \pm \mp)( \pm \mp) \ldots( \pm \mp)( \pm) .
$$


Here each of the pairs in parentheses can be $(+-)$ or $(-+)$, while the last sign is determined so that the total number of minuses is even. All such choices give the same $w \Lambda$, which is thus determined by the sequence $\epsilon_{1} \epsilon_{2} \ldots \epsilon_{n-2 k-1}$. We will therefore work only with $w$ of the form

$$
w=\epsilon_{1} \epsilon_{2} \ldots \epsilon_{n-2 k-1}(+-)(+-) \ldots(+-)( \pm),
$$

with the last sign determined as before. In other words, we choose the shortest possible $w$ in each case.

Theorem 5.2. The Dirac cohomology of $V_{k}$ is

$$
H_{D}\left(V_{k}\right)=\bigoplus_{w} E_{w \Lambda-\rho_{c}}
$$

with the summation over all $w$ as in (5.1). All ingredients of the formula (3.6) are uniquely determined by $w$ : $\sigma$ is given by

$$
\sigma=--\cdots-\epsilon_{1} \epsilon_{2} \ldots \epsilon_{n-2 k-1} \pm
$$

with the last sign determined so that the total number of minuses is even. The corresponding $K$-type in $V_{k}$ is the one with the highest weight of the form (2.4) given by

$$
d_{1}=\cdots=d_{k}=\text { the number of pluses in the sequence } \epsilon=\left(\epsilon_{1}, \ldots, \epsilon_{n-2 k-1}\right) .
$$

Proof. By (2.4), the lowest weights of the $K$-types of $V_{k}$ appearing in the right side of (3.6) are

$$
\left(0, \ldots, 0, d_{k}, d_{k}, \ldots, d_{1}, d_{1}\right)+(k, \ldots, k) .
$$

Since $(k, \ldots, k)$ is invariant under $W_{K}$, we can subtract it from both sides of (3.6). Now we can write out the new left side of (3.6) using the expression (2.3) for $\Lambda$ and (5.1) for $w$. To do this, let

$$
i_{1}>i_{2}>\cdots>i_{r}, \quad j_{1}<j_{2}<\cdots<j_{s}
$$

be the integers in $[1, n-2 k-1]$ such that

$$
w \Lambda=\left(i_{1}+k, \ldots, i_{r}+k, k, k-1, \ldots,-k,-j_{1}-k, \ldots,-j_{s}-k\right) .
$$

This means that $r+s=n-2 k-1$, and that

$$
\left\{i_{1}, \ldots, i_{r}, j_{1}, \ldots, j_{s}\right\}=\{1,2, \ldots, n-2 k-1\} .
$$

In terms of the sequence $\epsilon_{1}, \ldots, \epsilon_{n-2 k-1}$, the $i$ s and $j \mathrm{~s}$ are described by requiring $\epsilon_{n-2 k-i_{t}}=+, t=1, \ldots, r$, respectively $\epsilon_{n-2 k-j_{u}}=-, u=1, \ldots, s$. The sequence $\epsilon_{1}, \ldots, \epsilon_{n-2 k-1}$ is completely determined by the $i$ s and the $j$ s and vice versa. 
Now we see that

$$
\begin{aligned}
& w \Lambda-\rho_{c}+\rho_{n}-(k, \ldots, k) \\
& \quad=\left(i_{1}, i_{2}+1, \ldots, i_{r}+r-1, r, r, \ldots, r, r+1-j_{1}, r+2-j_{2}, \ldots, r+s-j_{s}\right) .
\end{aligned}
$$

Here $r$ appears in $2 k+1$ places, from the $(r+1)$-st place to the $(r+2 k+1)$-st. Note also that $r$ is the number of pluses in the sequence $\epsilon_{1}, \ldots, \epsilon_{n-2 k-1}$.

On the other hand, the right side of (3.6) becomes, after subtracting $(k, \ldots, k)$,

$$
\left(\sigma \rho-\rho_{c}+\rho_{n}+\left(0, \ldots, 0, d_{k}, d_{k}, \ldots, d_{1}, d_{1}\right)\right)^{\prime} .
$$

(Recall that the prime means taking the $\mathfrak{k}$-dominant $W_{K}$-conjugate.)

Since the largest component of (5.3) is $i_{1} \leq n-2 k-1$, we see that for (3.6) to hold, $\sigma \rho$ cannot have entries $n-1, n-2, \ldots, n-2 k$. Thus the starting $2 k$ signs of $\sigma$ are all minuses, that is,

$$
\sigma=-\cdots-\delta_{1} \ldots \delta_{n-2 k} .
$$

Note that $\delta_{n-2 k}$ is determined by the other $\delta$ s, because the number of minuses must be even.

Using similar notation as before, we see that

$$
\sigma \rho=\left(I_{1}, \ldots, I_{a}, 0,-J_{1}, \ldots,-J_{b},-(n-2 k),-(n-2 k+1), \ldots,-(n-1)\right),
$$

where $I_{1}>\cdots>I_{a}$ and $J_{1}<\cdots<J_{b}$ are integers in [1,n-2k-1] determined by $\delta_{1}, \ldots, \delta_{n-2 k-1}$. In particular, $a+b=n-2 k-1$,

$$
\left\{I_{1}, \ldots, I_{a}, J_{1}, \ldots, J_{b}\right\}=\{1,2, \ldots, n-2 k-1\},
$$

and $I \mathrm{~s}$ and $J \mathrm{~s}$ correspond to $\delta \mathrm{s}$ via

$$
\delta_{n-2 k-I_{u}}=+, \quad \delta_{n-2 k-J_{v}}=-,
$$

for all indices $u$ and $v$.

It is now easily seen that (5.4) equals

$$
\begin{aligned}
\left(I_{1}, I_{2}+1, \ldots, I_{a}+a-1,\right. & a, a+1-J_{1}, \\
& \left.a+2-J_{2}, \ldots, a+b-J_{b}, d_{k}, d_{k}, \ldots, d_{1}, d_{1}\right)^{\prime} .
\end{aligned}
$$

Thus $E_{w \Lambda-\rho_{c}}$ contributes to $H_{D}\left(V_{k}\right)$ if and only if (5.3) equals (5.6). Clearly, this is the case if $I \mathrm{~s}$ are equal to $i \mathrm{~s}, J \mathrm{~s}$ are equal to $j \mathrm{~s}$, and all $d \mathrm{~s}$ are equal to $r$. In this case, $\delta_{i}=\epsilon_{i}$ for $i=1, \ldots, n-2 k-1$. Thus to prove the theorem, we must show that this is the only possibility.

So let us assume that (5.3) equals (5.6). Either $i_{r}=1$ and $j_{1}>1$, or $i_{r}>1$ and $j_{1}=1$. The proof in each of these two cases is analogous, so we will assume that 
$i_{r}=1$ and $j_{1}>1$. Thus (5.3) is

$$
\left(i_{1}, \ldots, i_{r-1}+r-2, r, \ldots, r, r+1-j_{1}, \ldots, r+s-j_{s}\right) .
$$

Here the first $r+2 k+1$ entries are $\geq r$ while the last $s$ entries are $<r$. Moreover, at least $2 k+2$ entries are equal to $r$ - the entries starting with the $r$-th entry.

We claim that $a=r$. To see this, assume first that $a<r$. Then $a$ and $a+u-J_{u}$ for $u=1, \ldots, b$ are all $\leq a<r$. So there are at least $b+1=n-2 k-a$ entries $<r$ in (5.6), and therefore at most $2 k+a<2 k+r$ entries $\geq r$. But we saw that in (5.7) there are exactly $r+2 k+1$ entries are $\geq r$, so (5.7) (i.e., (5.3)) cannot equal (5.6).

Assume now that $a>r$. Then at least the first $a+1>r+1$ entries of (5.6) are $\geq a>r$, but the $(r+1)$-st entry of (5.7) is equal to $r$, so again (5.7) (i.e., (5.3)) cannot equal (5.6).

So we indeed see that $a=r$ and $b=s$. We now claim that $I_{r}=1$. Namely, if $I_{r}>1$, then the first $r$ entries of (5.6) are $>r$, but in (5.7) only the first $r-1$ entries can be $>r$. So indeed $I_{r}=1$ and hence $J_{1}>1$. This implies that $s$ entries of (5.6), $r+1-J_{1}, r+2-J_{2}, \ldots, r+s-J_{s}$, are $<r$. Since (5.7) has exactly $s$ entries that are $<r$, namely $r+1-j_{1}, \ldots, r+s-j_{s}$, we conclude that these entries must be equal, that is, that $J_{u}=j_{u}, u=1, \ldots, s$. It now follows that also $I_{t}=i_{t}, t=1, \ldots, r$, and finally that all $d \mathrm{~s}$ must be equal to $r$, as claimed in the theorem.

We leave it to the reader to formulate and prove analogs of Proposition 4.9 and Proposition 4.10.

\section{Some combinatorics of shuffles}

We now turn to some properties of shuffles, needed in the next section. An $(r, s)$ shuffle is a permutation

$$
i_{1}, \ldots, i_{r}, j_{1}, \ldots, j_{s}
$$

of the numbers $1,2, \ldots, r+s$ such that

$$
i_{1}<i_{2}<\cdots<i_{r}, \quad j_{1}<j_{2}<\cdots<j_{s} .
$$

To each such shuffle we associate the $(r+s)$-tuple $L=\left(L_{1}, \ldots, L_{r} \mid L_{1}^{\prime}, \ldots, L_{s}^{\prime}\right)$, where

$$
L_{u}=s+u-i_{u} \quad \text { and } \quad L_{v}^{\prime}=v-j_{v},
$$

for $u=1, \ldots, r$ and $v=1, \ldots, s$. Clearly,

$$
s \geq L_{1} \geq \cdots \geq L_{r} \geq 0 \geq L_{1}^{\prime} \geq \cdots \geq L_{s}^{\prime} \geq-r .
$$


Note also that it is easy to recover the shuffle from $L$ :

$$
i_{u}=s+u-L_{u} \quad \text { and } \quad j_{v}=v-L_{v}^{\prime},
$$

for $u=1, \ldots, r$ and $v=1, \ldots, s$. On the other hand, if we take an arbitrary $L$ satisfying (6.2), and calculate $i$ s and $j$ s as above, we will not necessarily obtain a shuffle. In that case $L$ is not attached to any shuffle.

Let $x \in\{0,1, \ldots, r\}$ be the unique index such that

$$
i_{x} \leq s \quad \text { but } \quad i_{x+1}>s .
$$

(If all $i_{u} \leq s$ we take $x=r$ and if all $i_{u}>s$ we take $x=0$.) Thus to get all numbers $1, \ldots, s$, besides $i_{1}, \ldots, i_{x}$ we must use $j_{1}, \ldots, j_{s-x}$. In other words,

$$
j_{s-x} \leq s \quad \text { and } \quad j_{s-x+1}>s .
$$

There are two cases: either $i_{x}=s$ and $j_{s-x}<s$, or $i_{x}<s$ and $j_{s-x}=s$. This immediately leads to the following lemma.

Lemma 6.3. If $x$ is as above, then exactly one of the following cases holds:

Case 1: $L_{x}=x, L_{s-x}^{\prime}>-x$

Case 2: $L_{x}>x, L_{s-x}^{\prime}=-x$

In each case, $L_{x} \geq x \geq L_{x+1}$, while $L_{s-x}^{\prime} \geq-x \geq L_{s-x+1}^{\prime}$.

One can characterize $x$ as the unique number in $\{0, \ldots, r\}$ such that $L_{x}=x$ or $L_{s-x}^{\prime}=-x$ (but not both). In this way, one can see what $x$ is directly from $L$.

Here is the result we are going to need in next section.

Proposition 6.4. Let $i_{1}, \ldots, i_{r}, j_{1}, \ldots, j_{s}$ be an $(r, s)$ shuffle and let

$$
L=\left(L_{1}, \ldots, L_{r} \mid L_{1}^{\prime}, \ldots, L_{s}^{\prime}\right)
$$

be attached to this shuffle as above. Let $x \in\{0, \ldots, r\}$ be as above. Let $M$ be an $(r+s)$-tuple obtained from $L$ by replacing one or several pairs of the form $a,-a$ by $x,-x$, and then rearranging to descending order. Then $M$ cannot be attached to a shuffle.

Proof. Assume that we are in Case 1, so $L_{x}=x$ and $L_{s-x}^{\prime}>-x$, while $L_{s-x+1}^{\prime} \leq$ $-x$. Note that after the changes we will still have $M_{x}=x$. If we were to replace any $(a,-a)$ with $a<x$ (and hence $-a>-x$ ), we would end up with $M_{s-x}=-x$. So Cases 1 and 2 happen simultaneously for $M$ and thus by Lemma 6.3, $M$ cannot be attached to a shuffle. So we can assume that all $a$ are $>x$. Let us choose $a$ to be the biggest possible, and let $u<x$ be the index such that $L_{u}=a$ and $L_{u+1}<a$.

It follows that

$i_{u}=s+u-L_{u}=s-a+u, \quad$ while $\quad i_{u+1}=s+u+1-L_{u+1}>s-a+u+1$. 
This implies that

$$
j_{s-a}<s-a+u, \quad \text { while } \quad j_{s-a+1}=s-a+u+1 .
$$

Namely, the numbers $1,2, \ldots, s-a+u$ include $i_{1}, \ldots, i_{u}$ and not $i_{u+1}$, hence they must also include $j_{1}, \ldots, j_{s-a}$. Also, $s-a+u+1$ is not among the $i$ s, hence it must be $j_{s-a+1}$.

This now tells us that

$$
L_{s-a}^{\prime}=s-a-j_{s-a}>-u, \quad \text { while } \quad L_{s-a+1}^{\prime}=s-a+1-j_{s-a+1}=-u .
$$

Since none of $L_{1}^{\prime}, \ldots, L_{s-x}^{\prime}$ is changed, and $s-a+1 \leq s-x$, we see that

$$
M_{s-a}^{\prime}=L_{s-a}^{\prime}>-u, \quad \text { while } \quad M_{s-a+1}^{\prime}=L_{s-a+1}^{\prime}=-u .
$$

On the other hand, since $L_{u}=a$ is replaced by $x$,

$$
M_{u}=L_{u+1}<a .
$$

We can now calculate $k_{c}=s+c-M_{c}, c=1, \ldots, r$ and $l_{d}=d-M_{d}, d=1, \ldots, s$, and check that $k_{1}, \ldots, k_{r}, l_{1}, \ldots, l_{s}$ is not a shuffle. Indeed, since $L_{u}$ was the first of the $L$ s that got changed, we see that

$$
k_{u-1}=i_{u-1}<i_{u}=s-a+u, \quad \text { while } k_{u}=s+u-M_{u}>s-a+u,
$$

so none of the $k_{c}$ s equals $s-a+u$. On the other hand,

$$
l_{s-a}=j_{s-a}<s-a+u, \quad \text { while } \quad l_{s-a+1}=j_{s-a+1}=s-a+u+1,
$$

so none of the $l_{d}$ s can be $s-a+u$ either.

The situation in Case 2 is analogous. We first use Lemma 6.3 to conclude that this time all replaced pairs $a,-a$ must satisfy $a<x$. We take the smallest (last) such $a$ and pick index $v$ such that $L_{v}=a$ while $L_{v-1}>a$. We then argue that the supposed shuffle attached to $M$ cannot contain the number $s-a+v$.

Remark 6.5. Using very similar reasoning as above it is not difficult to obtain the full conditions for an $L$ satisfying (6.2) to be attached to a shuffle. We omit this since Proposition 6.4 is all we need.

\section{The case of $u(p, q)$}

We are going to use the facts about the structure of the pair $(\mathfrak{g}, \mathfrak{k})$ which we reviewed in Section 2. Besides that, we also need to describe the subset $W^{1} \subset W_{G}$. In the unitary case, $W^{1}$ consists of $(p, q)$ shuffles, that is, each $w \in W^{1}$ is a permutation $i_{1}, \ldots, i_{p}, j_{1} \ldots, j_{q}$ of $1,2, \ldots, p+q$ such that $i_{1}<\cdots<i_{p}$ and $j_{1}<\cdots<j_{q}$.

The modules we are interested in are $V_{k}$, where $1 \leq k \leq \min (p, q)-1$ is an integer, of lowest weight $\mu_{0}=(k / 2, \ldots, k / 2 \mid-k / 2, \ldots,-k / 2)$. The modules $V_{k}$ 
were described in Section 2. In particular, the infinitesimal character $\Lambda$ of $V_{k}$ was given in (2.5). We rewrite $\Lambda$ as $\Lambda=\Lambda_{1}-\Lambda_{2}$, where

$$
\begin{aligned}
& \Lambda_{1}=\frac{1}{2}(p+q+1-k, p+q+1-k, \ldots, p+q+1-k), \\
& \Lambda_{2}=(1,2, \ldots, q-k, q-k+1, q-k+1, \ldots, q, q, q+1, q+2, \ldots, q+p-k) .
\end{aligned}
$$

We now want to write down (3.6) in our present case. We will first modify it by subtracting our lowest $K$-type $\mu_{0}$ from both sides. Since $\mu_{0}$ is $W_{K}$-invariant, it can be put inside the parentheses on the right side. To see what the left side is, note that $\Lambda_{1}$ is $W_{G}$-invariant, and calculate

$$
\Lambda_{1}-\rho_{c}+\rho_{n}-\mu_{0}=(q-k+1, q-k+2, \ldots, q-k+p \mid 1,2, \ldots, q) .
$$

Thus, after subtracting $\mu_{0}$ the left side of (3.6) becomes

$$
(q-k+1, q-k+2, \ldots, q-k+p \mid 1,2, \ldots, q)-w \Lambda_{2} .
$$

We are now going to apply Proposition 3.5, which says that $w \Lambda$ must be dominant regular for $\mathfrak{k}$. We already mentioned that $w \in W^{1}$, that is, $w$ is a $(p, q)$ shuffle. Regularity of $w \Lambda$ means that the first $p$ coordinates of $w \Lambda$ must be strictly decreasing, and so must the last $q$ coordinates. This means that $w$ must split apart each pair of equal coordinates in $\Lambda$. Hence we can take $w$ to be a permutation of the form

$$
\begin{array}{r}
w=\left(i_{1}, i_{2}, \ldots, i_{x}, q-k+1, q-k+3, \ldots, q+k-3, q+k-1, i_{x+k+1}, i_{x+k+2}, \ldots, i_{p}\right) \\
\left.j_{1}, j_{2}, \ldots, j_{y}, q-k+2, q-k+4, \ldots, q+k-2, q+k, j_{y+k+1}, j_{y+k+2}, \ldots, j_{q}\right) .
\end{array}
$$

Here

$$
\begin{array}{r}
1 \leq i_{1}<\cdots<i_{x} \leq q-k \\
1 \leq j_{1}<\cdots<j_{y} \leq q-k \\
q+k+1 \leq i_{x+k+1}<\cdots<i_{p} \leq p+q, \\
q+k+1 \leq j_{y+k+1}<\cdots<j_{q} \leq p+q,
\end{array}
$$

and all $i_{u}$ and $j_{v}$ are different integers. In particular, it follows that $i_{1}, \ldots, i_{x}$ and $j_{1}, \ldots, j_{y}$ exactly exhaust all numbers $1, \ldots, q-k$, and consequently $x+y=q-k$. (For each given $w \Lambda$, there are other choices for $w$ leading to the same $w \Lambda$, and the $w$ we choose is the shortest among them.)

It now follows that

$$
\begin{array}{r}
w \Lambda_{2}=\left(i_{1}, \ldots, i_{x}, q-k+1, q-k+2, \ldots, q, i_{x+k+1}-k, \ldots, i_{p}-k \mid\right. \\
\left.j_{1}, \ldots, j_{y}, q-k+1, q-k+2, \ldots, q, j_{y+k+1}-k, \ldots, j_{q}-k\right) .
\end{array}
$$


Plugging this into (7.1) we get for the left side of (3.6) the expression

$\left(q-k+1-i_{1}, \ldots, q-k+x-i_{x}, x, x, \ldots, x, q+k+x+1-i_{x+k+1}, \ldots, q+p-i_{p} \mid\right.$ $\left.1-j_{1}, \ldots, y-j_{y}, y-q+k, y-q+k, \ldots, y-q+k, y+k+1-j_{y+k+1}+k, \ldots, q-j_{q}+k\right)$.

There are $k$ components equal to $x$ in (7.3), and also $k$ components equal to $y-q+k=-x$.

To analyze the right side of (3.6), we first write

$$
\rho=\frac{1}{2}(p+q+1, p+q+1, \ldots, p+q+1)-(1,2, \ldots, p+q) .
$$

After a short calculation, this leads to

$$
\begin{aligned}
\sigma \rho-\rho_{c}+\rho_{n}=(q+1, q+2, \ldots, q+p \mid & 1,2, \ldots, q) \\
& -\sigma(1,2, \ldots, p \mid p+1, p+2, \ldots, p+q) .
\end{aligned}
$$

By (2.6), a general $K$-type of $V_{k}$ has lowest weight

$$
\mu_{0}+\left(0, \ldots, 0, d_{k}, \ldots, d_{1} \mid-d_{1}, \ldots,-d_{k}, 0, \ldots, 0\right),
$$

for some integers $d_{1} \geq d_{2} \geq \cdots \geq d_{k} \geq 0$. Thus, after subtracting $\mu_{0}$, the right side of (3.6) becomes

$$
\begin{aligned}
((q+1, \ldots, q+p & \mid 1, \ldots, q)-\sigma(1, \ldots, p \mid p+1, \ldots, p+q) \\
& \left.+\left(0, \ldots, 0, d_{k}, \ldots, d_{1} \mid-d_{1}, \ldots,-d_{k}, 0, \ldots, 0\right)\right)^{\prime}
\end{aligned}
$$

Now since $i_{1} \geq 1$, the first component of (7.3) is $\leq q-k$. Since (7.3) must be equal to (7.4), the first component of $\sigma(1, \ldots, p \mid p+1, \ldots, p+q)$ must be $\geq k+1$. (Namely, adding some $d_{u} \neq 0$ to a component can only make it larger.) This further implies that $\sigma(1, \ldots, p \mid p+1, \ldots, p+q)$ has $1, \ldots, k$ among the last $q$ coordinates.

Similarly, since $j_{q} \leq p+q$, the last component of (7.3) is $\geq-p+k$. Hence, the last component of $\sigma(1, \ldots, p \mid p+1, \ldots, p+q)$ must be $\leq p+q-k$. (Namely, subtracting some $d_{u} \neq 0$ from a component can only make it smaller.) This further implies that $\sigma(1, \ldots, p \mid p+1, \ldots, p+q)$ has $p+q-k+1, p+q-k+2, \ldots, p+q$ among its first $p$ components. Since $\sigma \in W^{1}$, we conclude that $\sigma(1, \ldots, p \mid p+1, \ldots, p+q)=$

$$
\left(I_{1}, \ldots, I_{p-k}, p+q-k+1, \ldots, p+q \mid 1, \ldots, k, J_{1}, \ldots, J_{q-k}\right),
$$

for some $k+1 \leq I_{1}<\cdots<I_{p-k} \leq p+q-k$ and $k+1 \leq J_{1}<\cdots<J_{q-k} \leq p+q-k$, such that all $I_{u}$ are different from all $J_{v}$; equivalently,

$$
\left\{I_{1}, \ldots, I_{p-k}, J_{1} \ldots, J_{q-k}\right\}=\{k+1, k+2, \ldots, p+q-k\} .
$$


Plugging this into (7.4), we get

$$
\begin{aligned}
& \left(q+1-I_{1}, \ldots, q+p-k-I_{p-k}, d_{k}, \ldots, d_{1} \mid\right. \\
& \left.-d_{1}, \ldots,-d_{k}, k+1-J_{1}, \ldots, q-J_{q-k}\right)^{\prime} .
\end{aligned}
$$

The question now is how we can make (7.5) equal to (7.3). Finding the answer to this question is equivalent to proving the following theorem.

Theorem 7.6. The Dirac cohomology of $V_{k}$ is

$$
H_{D}\left(V_{k}\right)=\bigoplus_{w} E_{w \Lambda-\rho_{c}},
$$

with the summation over all $w$ as in (7.2). All ingredients of the formula (3.6) are uniquely determined by $w$ :

The permutation

$$
\sigma=\left(I_{1}, \ldots, I_{p-k}, p+q-k+1, \ldots, p+q \mid 1, \ldots, k, J_{1}, \ldots, J_{q-k}\right)
$$

is given by

$$
\begin{array}{r}
\sigma=\left(i_{1}+k, \ldots, i_{x}+k, i_{x+k+1}-k, \ldots, i_{p}-k, p+q-k+1, \ldots, p+q \mid\right. \\
\left.1, \ldots, k, j_{1}+k, \ldots, j_{y}+k, j_{y+k+1}-k, \ldots, j_{q}-k\right),
\end{array}
$$

where $y=q-k-x$.

The corresponding $K$-type in $V_{k}$ is given by

$$
d_{1}=\cdots=d_{k}=x .
$$

Proof. We already know that for $E_{w \Lambda-\rho_{c}}$ to contribute to $H_{D}\left(V_{k}\right), w$ must be as in (7.2). Moreover, it is straightforward to check that if we choose $\sigma$ and $d_{1}, \ldots, d_{k}$ as in the statement of the theorem, then we do get equality of (7.5) and (7.3), and hence a contribution of $E_{w \Lambda-\rho_{c}}$ to $H_{D}\left(V_{k}\right)$.

It thus remains to show that there is no possible other choice of $I_{1}, \ldots, I_{p-k}$, $J_{1}, \ldots, J_{q-k}$ and $d_{1}, \ldots, d_{k}$ that would make the expressions (7.5) and (7.3) equal.

To see this, let us first relate (7.5) and (7.3) to shuffles. Let us set $r=p-k$, $s=q-k$, and define

$$
\begin{aligned}
& i_{u}^{\prime}=i_{u} \quad \text { for } u=1, \ldots, x, \quad i_{u}^{\prime}=i_{u+k}-2 k \quad \text { for } u=x+1, \ldots, r, \\
& j_{v}^{\prime}=j_{u} \text { for } u=1, \ldots, y, \quad j_{v}^{\prime}=j_{v+k}-2 k \text { for } u=x+1, \ldots, r \text {. }
\end{aligned}
$$

Then it is easy to check that $i_{1}^{\prime}, \ldots, i_{r}^{\prime}, j_{1}^{\prime}, \ldots, j_{s}^{\prime}$ is an $(r, s)$ shuffle, and the $(r+s)$ tuple $L_{1}$ associated to this shuffle as in (6.1) is exactly (7.3) with the $k$ terms $x$ and $k$ terms $-x$ omitted.

We further define

$$
I_{u}^{\prime}=I_{u}-k, \quad u=1, \ldots, r, \quad J_{v}^{\prime}=J_{v}-k, \quad v=1, \ldots, s .
$$


Then obviously $I_{1}^{\prime}, \ldots, I_{r}^{\prime}, J_{1}^{\prime}, \ldots, J_{s}^{\prime}$ is an $(r, s)$ shuffle, and the $(r+s)$-tuple $L_{2}$ associated to this shuffle as in (6.1) is exactly (7.5) with the terms $d_{k}, \ldots, d_{1}$ and $-d_{1}, \ldots,-d_{k}$ omitted.

Let us now suppose that (7.5) and (7.3) are equal, but not in the way stated in the theorem. This would mean that some of the $d_{i}$ are not equal to $x$, and consequently the corresponding $-d_{i}$ are not equal to $-x$. These pairs $d_{i},-d_{i}$ therefore have to appear in (7.3) with the terms $x$ and $-x$ omitted, that is in $L_{1}$. Then there must be an equal number of pairs $x,-x$ among the entries of (7.5) with the terms $d_{k}, \ldots, d_{1}$ and $-d_{1}, \ldots,-d_{k}$ omitted, that is, among the entries of $L_{2}$. The rest of $L_{2}$ must agree with the rest of $L_{1}$. Thus, $L_{2}$ is obtained from $L_{1}$ by taking several pairs of the form $a,-a$ and replacing them by $x,-x$. Since both $L_{1}$ and $L_{2}$ are obtained from shuffles, this is impossible by Proposition 6.4. This proves the theorem.

We leave it to the reader to formulate and prove analogs of Proposition 4.9 and Proposition 4.10.

\section{References}

[Adams 1987] J. Adams, "Unitary highest weight modules", Adv. in Math. 63:2 (1987), 113-137. MR 88b:22014 Zbl 0623.22014

[Adams 1994] J. Adams, "Nilpotent cohomology of the oscillator representation", J. Reine Angew. Math. 449 (1994), 1-7. MR 95f:22021 Zbl 0787.17019

[Enright 1988] T. J. Enright, “Analogues of Kostant's u-cohomology formulas for unitary highest weight modules”, J. Reine Angew. Math. 392 (1988), 27-36. MR 89m:22022 Zbl 0651.17003

[Enright and Willenbring 2004] T. J. Enright and J. F. Willenbring, "Hilbert series, Howe duality and branching for classical groups", Ann. of Math. (2) 159:1 (2004), 337-375. MR 2005d:22013 Zbl 1087.22011

[Enright et al. 1983] T. Enright, R. Howe, and N. Wallach, "A classification of unitary highest weight modules", pp. 97-143 in Representation theory of reductive groups (Park City, UT, 1982), edited by P. Trombi, Progress in Mathematics 40, Birkhäuser, Boston, 1983. MR 86c:22028 Zbl 0535.22012

[Howe 1989] R. Howe, "Remarks on classical invariant theory", Trans. Amer. Math. Soc. 313:2 (1989), 539-570. MR 90h:22015a Zbl 0674.15021

[Howe 1995] R. Howe, "Perspectives on invariant theory: Schur duality, multiplicity-free actions and beyond", pp. 1-182 in The Schur lectures (Tel Aviv, 1992), edited by I. Piatetski-Shapiro and S. Gelbart, Israel Math. Conf. Proc. 8, Bar-Ilan Univ., Ramat Gan, 1995. MR 96e:13006 Zbl 0844.20027

[Huang and Pandžić 2002] J.-S. Huang and P. Pandžić, "Dirac cohomology, unitary representations and a proof of a conjecture of Vogan", J. Amer. Math. Soc. 15:1 (2002), 185-202. MR 2002h:22022 Zbl 0980.22013

[Huang and Pandžić 2006] J.-S. Huang and P. Pandžić, Dirac operators in representation theory, Birkhäuser, Boston, 2006. MR 2007j:22025 Zbl 1103.22008

[Huang et al. 2006] J.-S. Huang, P. Pandžić, and D. Renard, "Dirac operators and Lie algebra cohomology”, Represent. Theory 10 (2006), 299-313. MR 2007k:22014 Zbl 1134.22011 
[Huang et al. 2009] J.-S. Huang, Y.-F. Kang, and P. Pandžić, "Dirac cohomology of some HarishChandra modules", Transform. Groups 14:1 (2009), 163-173. MR 2010g:22030 Zbl 1179.22013

[Kashiwara and Vergne 1978] M. Kashiwara and M. Vergne, "On the Segal-Shale-Weil representations and harmonic polynomials”, Invent. Math. 44:1 (1978), 1-47. MR 57 \#3311 Zbl 0375.22009

[Knapp 2002] A. W. Knapp, Lie groups beyond an introduction, 2nd ed., Progress in Mathematics 140, Birkhäuser, Boston, 2002. MR 2003c:22001 Zbl 1075.22501

[Kostant 1999] B. Kostant, "A cubic Dirac operator and the emergence of Euler number multiplets of representations for equal rank subgroups", Duke Math. J. 100:3 (1999), 447-501. MR 2001k:22032 Zbl 0952.17005

[Kostant 2003] B. Kostant, "Dirac cohomology for the cubic Dirac operator", pp. 69-93 in Studies in memory of Issai Schur (Chevaleret/Rehovot, 2000), edited by A. Joseph et al., Progr. Math. 210, Birkhäuser, Boston, 2003. MR 2004h:17005 Zbl 1165.17301

[Meng 2007] G. Meng, "MICZ-Kepler problems in all dimensions", J. Math. Phys. 48:3 (2007), 032105, 14. MR 2008f:81069 Zbl 1137.81325

[Meng 2008] G. Meng, "The representation aspect of the generalized hydrogen atoms", J. Lie Theory 18:3 (2008), 697-715. MR 2010h:81117 Zbl 1160.81029

[Meng 2010] G. Meng, "Generalized MICZ-Kepler problems and unitary highest weight modules, II”, J. Lond. Math. Soc. (2) 81:3 (2010), 663-678.

[Nishiyama et al. 2001] K. Nishiyama, H. Ochiai, and K. Taniguchi, "Bernstein degree and associated cycles of Harish-Chandra modules-Hermitian symmetric case", pp. 13-80 in Nilpotent orbits, associated cycles and Whittaker models for highest weight representations, Astérisque 273, Société Mathématique de France, 2001. MR 1845714 Zbl 0973.22008

[Pandžić 2004] P. Pandžić, "Coproducts for Clifford algebras”, Glas. Mat. Ser. III 39(59):2 (2004), 207-211. MR 2005h:11082

[Parthasarathy 1972] R. Parthasarathy, "Dirac operator and the discrete series", Ann. of Math. (2) 96 (1972), 1-30. MR 47 \#6945 Zbl 0249.22003

[Parthasarathy 1980] R. Parthasarathy, "Criteria for the unitarizability of some highest weight modules”, Proc. Indian Acad. Sci. Sect. A Math. Sci. 89:1 (1980), 1-24. MR 82c:22020 Zbl 0434.22011

[Parthasarathy et al. 1967] K. R. Parthasarathy, R. Ranga Rao, and V. S. Varadarajan, "Representations of complex semi-simple Lie groups and Lie algebras", Ann. of Math. (2) 85 (1967), 383-429. MR 37 \#1526

[Vergne and Rossi 1976] M. Vergne and H. Rossi, "Analytic continuation of the holomorphic discrete series of a semi-simple Lie group", Acta Math. 136:1-2 (1976), 1-59. MR 58 \#1032 Zbl 0356.32020

[Vogan 1997] J. Vogan, D. A., "Dirac operators and unitary representations", 3 talks at MIT Lie groups seminar, MIT, 1997.

[Wallach 1979] N. R. Wallach, "The analytic continuation of the discrete series, I, II", Trans. Amer. Math. Soc. 251 (1979), 1-17, 19-37. MR 81a:22009

[Wallach 1988] N. R. Wallach, Real reductive groups, I, Pure and Applied Mathematics 132, Academic Press, Boston, 1988. MR 89i:22029 Zbl 0666.22002

[Zhu and Huang 1997] C.-B. Zhu and J.-S. Huang, "On certain small representations of indefinite orthogonal groups”, Represent. Theory 1 (1997), 190-206. MR 98j:22026 Zbl 0887.22016

Received August 10, 2009. Revised April 15, 2010. 
JING-SONG HUANG

DEPARTMENT OF MATHEMATICS

Hong Kong University of SCIENCE AND TeChNOLOGY

Clear Water Bay, KowloON

HONG KONG SAR

CHINA

mahuang@ust.hk

PAVLE PANDŽIĆ

DEPARTMENT OF MATHEMATICS

UNIVERSITY OF ZAGREB

BIJENIČKA 30

10000 ZAGREB

CROATIA

pandzic@math.hr

VICTOR PROTSAK

DEPARTMENT OF MATHEMATICS

SUNY OSWEGO

7060 TOUTE 104

OSWEGO, NY 13126-3599

UNITED STATES

protsak@oswego.edu 


\title{
PACIFIC JOURNAL OF MATHEMATICS
}

\author{
http://www.pjmath.org \\ Founded in 1951 by
}

E. F. Beckenbach (1906-1982) and F. Wolf (1904-1989)

\section{EDITORS}

V. S. Varadarajan (Managing Editor)

Department of Mathematics

University of California

Los Angeles, CA 90095-1555

pacific@math.ucla.edu

Vyjayanthi Chari

Department of Mathematics

University of California

Riverside, CA 92521-0135

chari@math.ucr.edu

\section{Robert Finn}

Department of Mathematics Stanford University

Stanford, CA 94305-2125

finn@math.stanford.edu

Kefeng Liu

Department of Mathematics

University of California

Los Angeles, CA 90095-1555

liu@math.ucla.edu
Darren Long

Department of Mathematics

University of California

Santa Barbara, CA 93106-3080

long@math.ucsb.edu

Jiang-Hua Lu

Department of Mathematics

The University of Hong Kong

Pokfulam Rd., Hong Kong jhlu@maths.hku.hk

Alexander Merkurjev

Department of Mathematics University of California

Los Angeles, CA 90095-1555 merkurev@math.ucla.edu
Sorin Popa

Department of Mathematics

University of California

Los Angeles, CA 90095-1555

popa@math.ucla.edu

Jie Qing

Department of Mathematics

University of California

Santa Cruz, CA 95064

qing@ cats.ucsc.edu

Jonathan Rogawski

Department of Mathematics

University of California

Los Angeles, CA 90095-1555

jonr@math.ucla.edu

\section{PRODUCTION}

pacific@math.berkeley.edu

Silvio Levy, Scientific Editor Matthew Cargo, Senior Production Editor

ACADEMIA SINICA, TAIPEI

CALIFORNIA INST. OF TECHNOLOGY

INST. DE MATEMÁTICA PURA E APLICADA

KEIO UNIVERSITY

MATH. SCIENCES RESEARCH INSTITUTE

NEW MEXICO STATE UNIV.

OREGON STATE UNIV.

\section{SUPPORTING INSTITUTIONS}

STANFORD UNIVERSITY
UNIV. OF BRITISH COLUMBIA
UNIV. OF CALIFORNIA, BERKELEY
UNIV. OF CALIFORNIA, DAVIS
UNIV. OF CALIFORNIA, LOS ANGELES
UNIV. OF CALIFORNIA, RIVERSIDE
UNIV. OF CALIFORNIA, SAN DIEGO
UNIV. OF CALIF., SANTA BARBARA

UNIV. OF CALIF., SANTA CRUZ

UNIV. OF MONTANA

UNIV. OF OREGON

UNIV. OF SOUTHERN CALIFORNIA

UNIV. OF UTAH

UNIV. OF WASHINGTON

WASHINGTON STATE UNIVERSITY

These supporting institutions contribute to the cost of publication of this Journal, but they are not owners or publishers and have no responsibility for its contents or policies.

See inside back cover or www.pjmath.org for submission instructions.

The subscription price for 2011 is US \$420/year for the electronic version, and \$485/year for print and electronic.

Subscriptions, requests for back issues from the last three years and changes of subscribers address should be sent to Pacific Journal of Mathematics, P.O. Box 4163, Berkeley, CA 94704-0163, U.S.A. Prior back issues are obtainable from Periodicals Service Company, 11 Main Street, Germantown, NY 12526-5635. The Pacific Journal of Mathematics is indexed by Mathematical Reviews, Zentralblatt MATH, PASCAL CNRS Index, Referativnyi Zhurnal, Current Mathematical Publications and the Science Citation Index.

The Pacific Journal of Mathematics (ISSN 0030-8730) at the University of California, c/o Department of Mathematics, 969 Evans Hall, Berkeley, CA 94720-3840, is published monthly except July and August. Periodical rate postage paid at Berkeley, CA 94704, and additional mailing offices. POSTMASTER: send address changes to Pacific Journal of Mathematics, P.O. Box 4163, Berkeley, CA 94704-0163.

PJM peer review and production are managed by EditFLOW ${ }^{\mathrm{TM}}$ from Mathematical Sciences Publishers.

PUBLISHED BY PACIFIC JOURNAL OF MATHEMATICS

at the University of California, Berkeley 94720-3840

A NON-PROFIT CORPORATION

Typeset in IATEX

Copyright $(2011$ by Pacific Journal of Mathematics 


\section{PACIFIC JOURNAL OF MATHEMATICS}

Volume $250 \quad$ No. $1 \quad$ March 2011

Nonconventional ergodic averages and multiple recurrence for von $\quad 1$ Neumann dynamical systems

Tim Austin, TAnja EIsner and Terence TaO

Principal curvatures of fibers and Heegaard surfaces

WILLIAM BRESLIN

Self-improving properties of inequalities of Poincaré type on $s$-John domains

SENG-KeE CHUA and RICHARD L. WHEEDEN

The orbit structure of the Gelfand-Zeitlin group on $n \times n$ matrices

MARK COLARUSSO

On Maslov class rigidity for coisotropic submanifolds

VIKTOR L. GINZBURG

Dirac cohomology of Wallach representations

Jing-Song HuAng, PAVle PANDŽIĆ and Victor PROTSAK An example of a singular metric arising from the blow-up limit in the 191 continuity approach to Kähler-Einstein metrics

YALONG SHI and XIAOHUA ZHU

Detecting when a nonsingular flow is transverse to a foliation

SANDRA SHIELDS

Mixed interior and boundary nodal bubbling solutions for a sinh-Poisson equation

JUNCHENG WeI, LONG WEI and FENG ZHOU 